\title{
Curve Reconstruction, the Traveling Salesman Problem, and Menger's Theorem on Length*
}

\author{
J. Giesen \\ Institut für Theoretische Informatik, ETH Zürich, \\ CH-8092 Zürich, Switzerland \\ giesen@inf.eth2.ch
}

\begin{abstract}
We give necessary and sufficient regularity conditions under which the curve reconstruction problem is solved by a traveling salesman tour or path, respectively. For the proof we have to generalize a theorem of Menger [12], [13] on arc length.
\end{abstract}

\section{Introduction}

In 1930 Menger [12] proposed a new definition of arc length:

The length of an arc be defined as the least upper bound of the set of all numbers that could be obtained by taking each finite set of points of the curve and determining the length of the shortest polygonal graph joining all the points.

$\ldots$

We call this the messenger problem (because in practice the problem has to be solved by every postman, and also by many travelers): finding the shortest path joining all of a finite set of points, whose pairwise distances are known.

This statement is one of the first references to the Traveling Salesman Problem.

Arc length is commonly defined as the least upper bound of the set of numbers obtained by taking each finite set of points of the curve and determining the length of the polygonal graph joining all the points in their order along the arc. In [13] Menger proves the equivalence of his definition and the common one (Menger's theorem).

It seems natural to think that this equivalence holds due to the fact that the shortest polygonal graph coincides with the polygonal graph joining the points in their order along

\footnotetext{
* This work was supported by grants from the Swiss Federal Office for Education and Science (Projects ESPRIT IV LTR No. 21957 CGAL and No. 28155 GALIA).
} 
the arc, provided the set of points is sufficiently dense. In other words, for sufficiently dense point sets a traveling salesman path solves the polygonal reconstruction problem for arcs. This problem was stated by Amenta et al. [4] as follows:

Given a curve $\gamma \in \mathbb{R}^{d}$ and a finite set of sample points $S \subset \gamma$. A polygonal reconstruction of $\gamma$ from $S$ is a graph that connects every pair of samples adjacent along $\gamma$, and no others.

However, Menger's proof does not show this at all. So we want to study the question whether the polygonal reconstruction problem is solved by a traveling salesman path, provided the sample points are sufficiently dense in the curve. Since a traveling salesman path is always simple, we cannot expect that it solves the reconstruction problem for curves with intersections. Even worse, the traveling salesman path may not coincide with the polygonal reconstruction for arbitrarily dense samples of simple curves. Consider the following example:

Let $\gamma$ be the simple arc which consists of the unit interval on the $x$-axis and the graph of $y=x^{2}$ on this interval. That is,

$$
\gamma:[0,1] \rightarrow \mathbb{R}^{2}, t \mapsto \begin{cases}(1-2 t, 0), & t \leq \frac{1}{2}, \\ \left(2 t-1,(2 t-1)^{2}\right), & t>\frac{1}{2}\end{cases}
$$

For large $n$ the samples

$$
S_{n}=\left\{p_{n}^{1}, p_{n}^{2}, p_{n}^{3}, p_{n}^{4}\right\} \cup \bigcup_{i=2}^{n}\left\{\left(\frac{i}{n}, 0\right),\left(\frac{i}{n}, \frac{i^{2}}{n^{2}}\right)\right\}
$$

with

$$
\begin{array}{ll}
p_{n}^{1}=\left(\frac{1}{n}, 0\right), & p_{n}^{2}=\left(\frac{1}{n^{3}}, \frac{1}{n^{6}}\right), \\
p_{n}^{3}=\left(\frac{2}{n^{3}}, \frac{4}{n^{6}}\right), & p_{n}^{4}=\left(\frac{1}{n^{2}}, \frac{1}{n^{4}}\right)
\end{array}
$$

become arbitrarily dense in $\gamma$. However, the traveling salesman path through $S_{n}$ is different from the polygonal reconstruction from $S_{n}$ (see Fig. 1) because

$$
\left|p_{n}^{1}-p_{n}^{2}\right|+\left|p_{n}^{2}-p_{n}^{3}\right|+\left|p_{n}^{3}-p_{n}^{4}\right|>\left|p_{n}^{1}-p_{n}^{3}\right|+\left|p_{n}^{3}-p_{n}^{2}\right|+\left|p_{n}^{2}-p_{n}^{4}\right| .
$$

In this example, the arc $\gamma$ has finite length and finite total absolute curvature. Thus, even finite curvature, which is a stronger property than finite length, see [1], is not sufficient for the polygonal reconstruction problem to be solved by a traveling salesman path, provided the points are sampled densely enough. The crucial point is that $\gamma$ behaves quite well, but is not regular at $(0,0) \in \gamma$. The regularity conditions necessary turn out to be only slightly stronger.

In this paper we prove:

Suppose that for every point of a simple curve:

1. The left and the right tangents exist and are nonzero.

2. The smaller angle between these tangents is less than $\pi$.

Under these conditions there exists a finite sampling density such that the traveling 


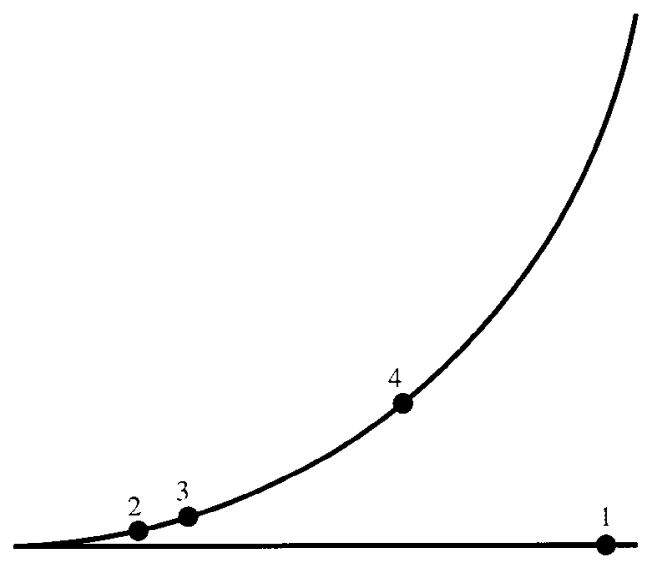

Fig. 1. 1-3-2-4 is shorter than 1-2-3-4.

salesman path solves the polygonal reconstruction problem for all samples with larger sample density.

Regularity is a local property. In contrast to that, it is a global property for a path to be a shortest polygonal path through a finite point set. One of the most interesting aspects of our work is this transition from a local to a global property and the methods used therein. We first prove a local version of our global theorem by using a projection technique from integral geometry. We believe that this technique could be useful in many other contexts, even in the study of higher-dimensional objects than curves. At a first glance it is not obvious how to derive the global version from the local one. This extension is achieved by an application of two corollaries of Menger's theorem. As a by-product we generalize Menger's theorem to simple closed curves in Euclidean space.

\section{Basic Definitions}

In this section we give definitions and cite theorems that we need for our proofs. The results that we present are only valid for connected simple curves in Euclidean space. Hence in the following $\gamma:[0,1] \rightarrow \mathbb{R}^{d}$ is always a connected simple curve. We use the symbol $\gamma$ either to denote a mapping or to denote the image of the mapping. It should always be clear from the context what $\gamma$ denotes.

We call a curve $\gamma \mathrm{s}(\mathrm{emi})$-regular if in every point on $\gamma$ nonzero left and right tangents exist. This is expressed in the following definition:

Definition 2.1. Let

$$
T=\left\{\left(t_{1}, t_{2}\right): t_{1}<t_{2}, t_{1}, t_{2} \in[0,1]\right\}
$$

and

$$
\tau: T \rightarrow \mathbb{S}^{d-1},\left(t_{1}, t_{2}\right) \mapsto \frac{\gamma\left(t_{2}\right)-\gamma\left(t_{1}\right)}{\left|\gamma\left(t_{2}\right)-\gamma\left(t_{1}\right)\right|}
$$


The curve $\gamma$ is called left (right) regular at $\gamma\left(t_{0}\right)$ with left tangent $1\left(\gamma\left(t_{0}\right)\right)$ or right tangent $\mathrm{r}\left(\gamma\left(t_{0}\right)\right)$ if for every sequence $\left(\xi_{n}\right)$ in $T$ which converges from the left (right) to $\left(t_{0}, t_{0}\right)$ in closure $(T)$ the sequence $\tau\left(\xi_{n}\right)$ converges to $1\left(\gamma\left(t_{0}\right)\right)$ or $\mathrm{r}\left(\gamma\left(t_{0}\right)\right)$, respectively. We call $\gamma$ s-regular if it is left and right regular in all points $\gamma(t), t \in[0,1]$. A curve is called regular if it is s-regular and in every point left and right tangent coincide.

The relationship between s-regularity and two of the most interesting geometric properties of curves, length and total absolute curvature, was shown by Aleksandrov and Reshetnyak [1].

Theorem 2.1. Every curve $\gamma$ of finite total absolute curvature $C(\gamma)$ is $s$-regular and every s-regular curve has finite length $L(\gamma)$.

Both length and total absolute curvature are defined via inscribed polygons. For the definition of inscribed polygons we need the definition of a sample, which we give next.

Definition 2.2. A sample $S$ of $\gamma$ is a finite sequence

$$
S=\left\{p^{1}, \ldots, p^{|S|}\right\}
$$

of points where $p^{i} \in \gamma$. We assume that the sample points $p^{i}$ are ordered according to the order of the $\gamma^{-1}\left(p^{i}\right) \in[0,1]$. To every sample $S$ its density is defined to be the inverse of the following number:

$$
\varepsilon(S)=\sup _{x \in \gamma} \min \left\{\left|p^{i}-x\right|: i=0, \ldots, n\right\} .
$$

In the following we sometimes make use of the convention $p^{|S|+1}=p^{|S|}$ if $\gamma$ is open and $p^{|S|+1}=p^{1}$ if $\gamma$ closed.

Sometimes, especially for closed curves, it is more appropriate to make use of an ordering of the sample points that is independent of a specific parameterization.

Definition 2.3. Let $S=\left\{p^{1}, \ldots, p^{|S|}\right\}$ be a sample of $\gamma$. If $\gamma$ is open one writes $i \triangleleft j$ if $\gamma^{-1}\left(p^{i}\right)<\gamma^{-1}\left(p^{j}\right)$. This notion is independent of an orientation preserving change of parameterization. If $\gamma$ is closed and has finite length choose an orientation along $\gamma$ and write $i \triangleleft j$ if $i \neq j$ and $L\left(\gamma\left(p^{i}: p^{j}\right)\right) \leq L\left(\gamma\left(p^{j}: p^{i}\right)\right)$, where $\gamma\left(p^{i}: p^{j}\right) \subset \gamma$ is the arc connecting $p^{i}$ and $p^{j}$ in the orientation along $\gamma$. One writes $i \unlhd j$ if the possibility that $i=j$ is included.

Now we are prepared to give the definition of length and total absolute curvature. Let $P(S)$ denote the polygon that connects a sample $S$ in the right order.

\section{Definition 2.4.}

1. The (Jordan) length $L(\gamma)$ of a curve $\gamma$ is the following number: $\sup \{L(P(S)): S$ is a sample of $\gamma\}$, 
where

$$
L(P(S))=\sum_{i=1}^{|S|}\left|p^{i+1}-p^{i}\right| .
$$

2. The curvature $C(\gamma)$ of a curve $\gamma$ is defined as the number

$$
\sup \{C(P(S)): S \text { is a sample of } \gamma\},
$$

where

$$
C(P(S))=\sum_{i=1}^{|S|-2}\left|\angle\left(p^{i+1}-p^{i}, p^{i+2}-p^{i+1}\right)\right|,
$$

if $\gamma$ is open, and

$$
C(P(S))=\sum_{i=1}^{|S|-1}\left|\angle\left(p^{i+1}-p^{i}, p^{i+2}-p^{i+1}\right)\right|,
$$

where $p^{|S|+1}=p^{1}$, if $\gamma$ is closed.

In the following we make use of a theorem of Reshetnyak [14], which states how one gets the length of a curve by integration over the length of the orthogonal projections of the curve on all one-dimensional subspaces $\ell \in \mathbb{R}^{d+1}$. The collection of all one-dimensional subspaces of $\mathbb{R}^{d+1}$ forms the $d$-dimensional projective space $\mathbb{P}^{d}$. In the following the elements of $\mathbb{P}^{d}$ are sometimes called lines. From a standard construction in integral geometry one gets a probability measure $\mu_{d}$ on $\mathbb{P}^{d}$ from the surface area measure on the $d$-dimensional sphere $\mathbb{S}^{d}$ :

The continuous and locally homeomorphic mapping

$$
\varphi: \mathbb{S}^{d}=\left\{x \in \mathbb{R}^{d+1}:|x|=1\right\} \rightarrow \mathbb{P}^{d}, \quad x \mapsto\{\lambda x: \lambda \in \mathbb{R}\},
$$

is a double cover. Since $\varphi$ is continuous, it is also measurable. That is the inverse image $\varphi^{-1}(B)$ of a Borel set $B \in \mathbb{P}^{d}$ is a Borel set in $\mathbb{S}^{d}$. On $\mathbb{S}^{d}$ we have the usual surface area measure $v_{d}$. Therefore we get a measure $\mu_{d}^{\prime}$ on $\mathbb{P}^{d}$ as $\mu_{d}^{\prime}(B)=v_{d}\left(\varphi^{-1}(B)\right)$ for all Borel sets $B \subseteq \mathbb{P}^{d}$. We have

$$
\int_{\mathbb{P}^{d}} \mathrm{~d} \mu_{d}^{\prime}=\operatorname{vol}\left(\mathbb{S}^{d}\right)=\frac{2 \pi^{(d+1) / 2}}{\Gamma((d+1) / 2)},
$$

which we use to normalize $\mu_{d}^{\prime}$. That is, we set

$$
\mu_{d}(B)=\frac{1}{\operatorname{vol}\left(\mathbb{S}^{d}\right)} \mu_{d}^{\prime}(B)
$$

for all Borel sets $B \subset \mathbb{P}^{d}$.

The theorem of Reshetnyak now reads as follows:

Theorem 2.2. For any s-regular curve $\gamma$ one finds

$$
\int_{\mathbf{P}^{d}} L\left(\pi_{\ell}(\gamma)\right) \mathrm{d} \mu_{d}(\ell)=c_{d} L(\gamma) \quad \text { with } \quad c_{d}=\frac{\Gamma((d+1) / 2)}{\sqrt{\pi} \Gamma((d+2) / 2)} .
$$




\section{A Local Analysis}

In this section we give several reformulations of our notion of regularity. We end up with a reformulation which is a local version of the theorem we want to prove in this paper. The main tool to prove this version is Theorem 2.2. To apply this theorem we have to study projections of sample points on one-dimensional subspaces.

The first reformulation of regularity in the following lemma is a pure metric interpretation of our definition of regularity.

Lemma 3.1. Let $\gamma$ be a simple closed curve, which is left (right) regular in $p \in \gamma$. Let $\left(p_{n}\right),\left(q_{n}\right)$, and $\left(r_{n}\right)$ be sequences of points from $\gamma$, that converge to $p$ from the left (right), such that $p_{n}<q_{n}<r_{n}$ for all $n \in \mathbb{N}$ in an order locally around $p$ along $\gamma$. Let $\alpha_{n}$ be the angle at $q_{n}$ of the triangle with corner points $p_{n}, q_{n}$, and $r_{n}$. Then the sequence of angles $\left(\alpha_{n}\right)$ converges to $\pi$.

Proof. Since $\gamma$ seen as a mapping from an interval is locally a homeomorphism the sequences $\left(\gamma^{-1}\left(p_{n}\right)\right),\left(\gamma^{-1}\left(q_{n}\right)\right)$, and $\left(\gamma^{-1}\left(r_{n}\right)\right)$ converge to $\gamma^{-1}(p)$. Thus by our definition of left (right) regularity asymptotically the three oriented segments

$$
\operatorname{conv}\left\{p_{n}, q_{n}\right\}, \operatorname{conv}\left\{q_{n}, r_{n}\right\} \text {, and } \operatorname{conv}\left\{p_{n}, r_{n}\right\}
$$

have to point in the direction of the left (right) tangent $1(p)$ or $\mathrm{r}(p)$, respectively. That is, $\lim _{n \rightarrow \infty} \alpha_{n}=\pi$.

We believe that this metric aspect of regularity is the most important one for our theorem to hold, but in the following we have to make use of the linear structure of $\mathbb{R}^{d+1}$. We exploit this linear structure by studying projections of a set of sample points on one-dimensional subspaces of $\mathbb{R}^{d+1}$.

In the following let $\gamma$ be s-regular and let $p \in \gamma$ be a fixed point. We want to introduce the following notions. Let $\eta>0$. The connected component of

$$
\{q \in \gamma:|p-q|<\eta\}
$$

which contains $p$ is denoted by $B_{\eta}(p)$. We assume that every one-dimensional subspace $\ell$ of $\mathbb{R}^{d+1}$ not perpendicular to $l(p)$ is oriented according to the orientation induced by the orthogonal projection $\pi_{\ell}(1(p))$ of $1(p)$ on $\ell$ and that every one-dimensional subspace $\ell$ perpendicular to $l(p)$ carries an arbitrary orientation.

We want to compare the ordering of a set of sample points close to $p$ on $\gamma$ with the ordering of the projections of these points on one-dimensional subspaces of $\mathbb{R}^{d+1}$. The following reformulation of regularity states to which extent these orderings can be different:

Lemma 3.2. Assume there exists a sequence $\left(\ell_{n}\right)$ of one-dimensional subspaces of $\mathbb{R}^{d+1}$ and sequences $\left(p_{n}\right),\left(q_{n}\right),\left(r_{n}\right) \in B_{1 / n}(p)$ with $p_{n}<q_{n}<r_{n} \leq p$ in the order along $\gamma$, but

$$
\pi_{\ell_{n}}\left(q_{n}\right)<\pi_{\ell_{n}}\left(p_{n}\right)<\pi_{\ell_{n}}\left(r_{n}\right) \quad \text { or } \quad \pi_{\ell_{n}}\left(p_{n}\right)<\pi_{\ell_{n}}\left(r_{n}\right)<\pi_{\ell_{n}}\left(q_{n}\right)
$$


in the order on $\ell_{n}$. Then the limit of every convergent subsequence of $\left(\ell_{n}\right)$ has to be perpendicular to $1(p)$.

Proof. We want to do the proof by contradiction. That is, we can assume that $\left(\ell_{n}\right)$ converges in the topology of $\mathbb{P}^{d}$ and that

$$
0 \leq \beta:=\lim _{n \rightarrow \infty} \angle\left(1(p), \ell_{n}\right)<\frac{\pi}{2},
$$

where $\angle\left(1(p), \ell_{n}\right)$ denotes the smaller of the two angles defined by the subspace $\ell_{n}$ and the subspace spanned by $1(p)$. For the proof we assume without loss of generality that $\pi_{\ell_{n}}\left(q_{n}\right)<\pi_{\ell_{n}}\left(p_{n}\right)<\pi_{\ell_{n}}\left(r_{n}\right)$. Furthermore, we can assume by moving $q_{n}$ or $r_{n}$ a little bit on $\gamma$ that there exists $0<\lambda<1$ (independent of $n$ ) such that

$$
\left|\pi_{\ell_{n}}\left(p_{n}\right)-\pi_{\ell_{n}}\left(r_{n}\right)\right|=\lambda \cos (\beta)\left|\pi_{\ell_{n}}\left(q_{n}\right)-\pi_{\ell_{n}}\left(r_{n}\right)\right| .
$$

Why can we do so? By our assumption we have

$$
\left|\pi_{\ell_{n}}\left(q_{n}\right)-\pi_{\ell_{n}}\left(r_{n}\right)\right|=\left|\pi_{\ell_{n}}\left(q_{n}\right)-\pi_{\ell_{n}}\left(p_{n}\right)\right|+\left|\pi_{\ell_{n}}\left(p_{n}\right)-\pi_{\ell_{n}}\left(r_{n}\right)\right|,
$$

and hence

$$
\left|\pi_{\ell_{n}}\left(p_{n}\right)-\pi_{\ell_{n}}\left(r_{n}\right)\right|=\lambda_{n}\left|\pi_{\ell_{n}}\left(q_{n}\right)-\pi_{\ell_{n}}\left(r_{n}\right)\right|
$$

with $0<\lambda_{n}<1$. From the continuity of $\gamma$ and the continuity of the projection maps $\pi_{\ell_{n}}$ we find that moving $p_{n}$ toward $q_{n}$ on $\gamma$ can make $\left|\pi_{\ell_{n}}\left(q_{n}\right)-\pi_{\ell_{n}}\left(p_{n}\right)\right|$ arbitrarily small. Similarly, moving $r_{n}$ toward $q_{n}$ on $\gamma$ can make $\left|\pi_{\ell_{n}}\left(p_{n}\right)-\pi_{\ell_{n}}\left(r_{n}\right)\right|$ arbitrarily small. Now move $p_{n}$ toward $q_{n}$ on $\gamma$ if $\lambda_{n}<\lambda \cos (\beta)$ or move $r_{n}$ toward $q_{n}$ on $\gamma$ if $\lambda_{n}>\lambda \cos (\beta)$ as long as $\lambda_{n} \neq \lambda \cos (\beta)$.

From the regularity of $\gamma$ we find that

$$
\lim _{n \rightarrow \infty} \angle\left(\operatorname{conv}\left\{q_{n}, r_{n}\right\}, 1(p)\right)=\lim _{n \rightarrow \infty} \angle\left(\operatorname{conv}\left\{p_{n}, r_{n}\right\}, 1(p)\right)=0
$$

and together with the triangle inequality for spherical triangles

$$
\begin{aligned}
\lim _{n \rightarrow \infty} \angle & \left(\operatorname{conv}\left\{q_{n}, r_{n}\right\}, \pi_{\ell_{n}}\left(\operatorname{conv}\left\{q_{n}, r_{n}\right\}\right)\right) \\
& \leq \lim _{n \rightarrow \infty}\left(\angle\left(\operatorname{conv}\left\{q_{n}, r_{n}\right\}, 1(p)\right)+\angle\left(1(p), \pi_{\ell_{n}}\left(\operatorname{conv}\left\{q_{n}, r_{n}\right\}\right)\right)\right) \\
& =\lim _{n \rightarrow \infty} \angle\left(\operatorname{conv}\left\{q_{n}, r_{n}\right\}, 1(p)\right)+\lim _{n \rightarrow \infty} \angle\left(1(p), \pi_{\ell_{n}}\left(\operatorname{conv}\left\{q_{n}, r_{n}\right\}\right)\right) \\
& =0+\lim _{n \rightarrow \infty} \angle\left(1(p), \ell_{n}\right) \\
& =\beta<\frac{\pi}{2} .
\end{aligned}
$$

Analogously we find

$$
\lim _{n \rightarrow \infty} \angle\left(\operatorname{conv}\left\{p_{n}, r_{n}\right\}, \pi_{\ell_{n}}\left(\operatorname{conv}\left\{p_{n}, r_{n}\right\}\right)\right) \leq \beta<\frac{\pi}{2} .
$$

Hence

$$
0<\cos (\beta) \leq \lim _{n \rightarrow \infty} \frac{\left|\pi_{\ell_{n}}\left(q_{n}\right)-\pi_{\ell_{n}}\left(r_{n}\right)\right|}{\left|q_{n}-r_{n}\right|} \leq 1
$$


and

$$
0<\cos (\beta) \leq \lim _{n \rightarrow \infty} \frac{\left|\pi_{\ell_{n}}\left(p_{n}\right)-\pi_{\ell_{n}}\left(r_{n}\right)\right|}{\left|p_{n}-r_{n}\right|} \leq 1 .
$$

Therefrom we find

$$
\begin{aligned}
\frac{1}{\cos (\beta)} & \geq \lim _{n \rightarrow \infty} \frac{\left|\pi_{\ell_{n}}\left(q_{n}\right)-\pi_{\ell_{n}}\left(r_{n}\right)\right|}{\left|q_{n}-r_{n}\right|} \frac{\left|p_{n}-r_{n}\right|}{\left|\pi_{\ell_{n}}\left(p_{n}\right)-\pi_{\ell_{n}}\left(r_{n}\right)\right|} \\
& =\lim _{n \rightarrow \infty} \frac{\left|\pi_{\ell_{n}}\left(q_{n}\right)-\pi_{\ell_{n}}\left(r_{n}\right)\right|}{\left|\pi_{\ell_{n}}\left(p_{n}\right)-\pi_{\ell_{n}}\left(r_{n}\right)\right|} \frac{\left|p_{n}-r_{n}\right|}{\left|q_{n}-r_{n}\right|} \\
& =\lim _{n \rightarrow \infty} \frac{\left|\pi_{\ell_{n}}\left(q_{n}\right)-\pi_{\ell_{n}}\left(r_{n}\right)\right|}{\left|\pi_{\ell_{n}}\left(p_{n}\right)-\pi_{\ell_{n}}\left(r_{n}\right)\right|} \lim _{n \rightarrow \infty} \frac{\left|p_{n}-r_{n}\right|}{\left|q_{n}-r_{n}\right|} \\
& =\frac{1}{\lambda \cos (\beta)} \lim _{n \rightarrow \infty} \frac{\left|p_{n}-r_{n}\right|}{\left|q_{n}-r_{n}\right|}
\end{aligned}
$$

and thus

$$
\lim _{n \rightarrow \infty} \frac{\left|p_{n}-r_{n}\right|}{\left|q_{n}-r_{n}\right|} \leq \lambda<1
$$

Hence there exists $N \in \mathbb{N}$ such that $\left|p_{n}-r_{n}\right| \leq\left|q_{n}-r_{n}\right|$ for all $n \geq N$. Now we consider the triangles with corner points $p_{n}, q_{n}$, and $r_{n}$. Let $\alpha_{n}$ be the angle at $q_{n}$. From the law of cosines together with $\left|p_{n}-r_{n}\right| \leq\left|q_{n}-r_{n}\right|$ we find

$$
\cos \left(\alpha_{n}\right)=-\frac{\left|p_{n}-r_{n}\right|^{2}-\left|p_{n}-q_{n}\right|^{2}-\left|q_{n}-r_{n}\right|^{2}}{2\left|p_{n}-q_{n}\right|\left|q_{n}-r_{n}\right|}>0 .
$$

Thus $\alpha_{n}$ has to be smaller than or equal to $\pi / 2$ for all $n \geq N$. Since $\gamma$ is left regular in $p$ that is a contradiction to Lemma 3.1 .

From this lemma we can derive another one that states the measure $\mu_{d}$ of the set of lines on which the sample points in a small neighborhood left from $p$ are not projected in the right order turns to zero as the neighborhood shrinks to $p$.

Lemma 3.3. For all $n \in \mathbb{N}$ let $p_{n}, q_{n}, r_{n} \in B_{1 / n}(p)$ with $p_{n}<q_{n}<r_{n} \leq p$ in the order along $\gamma$. Let

$$
\begin{gathered}
N_{n}=\left\{\ell \in \mathbb{P}^{d}: \pi_{\ell}\left(q_{n}\right)<\pi_{\ell}\left(p_{n}\right)<\pi_{\ell}\left(r_{n}\right) \text { or } \pi_{\ell}\left(p_{n}\right)<\pi_{\ell}\left(r_{n}\right)\right. \\
\left.<\pi_{\ell}\left(q_{n}\right) \text { in the order on } \ell\right\},
\end{gathered}
$$

where $\pi_{\ell}$ denotes the orthogonal projection on the line $\ell$. Then $\lim _{n \rightarrow \infty} \mu_{d}\left(N_{n}\right)=0$.

Proof. In [14] Reshetnyak shows: Let $V$ be a $d$-dimensional subspace of $\mathbb{R}^{d+1}$ and let $E \subset \mathbb{P}^{d}$ be the set of all one-dimensional subspaces contained in $V$. Then $\mu_{d}(E)=$ 0 . The proof of the lemma follows directly from this theorem of Reshetnyak and Lemma 3.2. 
Obviously an analogous result for sample points larger than $p$ in the order along $\gamma$ also holds.

Now we are prepared to prove a local version of our theorem. For a given sample $S$ of a neighborhood of $p$ we fix the smallest and the largest sample point along $\gamma$ and consider paths through $S$ which connect these points. One such path is the polygonal reconstruction $P(S)$ which connects the sample points in their order along $\gamma$. We distinguish three types of lines $\ell \in \mathbb{P}^{d}$ :

1. There exists a path through the sample points different from $P(S)$ which has a shorter projection on $\ell$ than $P(S)$.

2. Every path through the sample points different from $P(S)$ has a larger projection on $\ell$ than $P(S)$.

3. There exist paths through the sample points different from $P(S)$ which has projections on $\ell$ with the same length as the projection of $P(S)$, but there is no path with a shorter projection.

The proof is subdivided into three steps. First, we show that the measure of the first set of lines tends to zero as the neighborhood of $p$ shrinks to $p$ itself. Second, there exists a constant larger than zero such that the measure of the second set of lines is larger than this constant for arbitrary small neighborhoods of $p$. In the third step we want to apply Theorem 2.2. We use this theorem and the results of the first two steps to show that for small neighborhoods of $p$ the polygonal reconstruction $P(S)$ has to be the shortest path through the sample points.

Theorem 3.1. Assume

$$
\alpha=\sup \{\angle(1(q), \mathrm{r}(q)): q \in \gamma\}<\pi
$$

and let $\left(S_{n}\right)$ be a sequence of samples of $B_{1 / n}(p)$. Let $\operatorname{TSP}^{*}\left(S_{n}\right)$ be a path of minimal length through the sample points $S_{n}$ with fixed startpoint $\min S_{n}$ and fixed endpoint $\max S_{n}$. Here min and $\max$ are taken with respect to the order induced on $S_{n}$ by $\gamma$. Then there exists $N \in \mathbb{N}$ such that

$$
T S P^{*}\left(S_{n}\right)=P\left(S_{n}\right),
$$

for all $n \geq N$. Furthermore, $\operatorname{TSP}^{*}\left(S_{n}\right)$ is unique for all $n \geq N$.

Proof. First Step. Let $L_{n} \subset \mathbb{P}^{d}$ be the set of lines for which the projection $\pi_{\ell}\left(P\left(S_{n}\right)\right)$ is not a shortest path through the projected sample points $\pi_{\ell}\left(S_{n}\right)$ and let $\mu_{d}$ be the probability measure on $\mathbb{P}^{d}$ introduced in (1).

We show that

$$
\lim _{n \rightarrow \infty} \mu_{d}\left(L_{n}\right)=0 .
$$

For the proof we use the following abbreviations (min and max denote the minimum and the maximum along $\gamma$ and $\min _{\ell}$ and $\max _{\ell}$ denote the minimum and the maximum along $\ell$ ):

$$
m_{1}=\pi_{\ell}\left(\min S_{n}\right), \quad m_{2}=\min _{\ell} \pi_{\ell}\left(S_{n}\right), \quad m_{3}=\max _{\ell} \pi_{\ell}\left(S_{n}\right), \quad m_{4}=\pi_{\ell}\left(\max S_{n}\right) .
$$




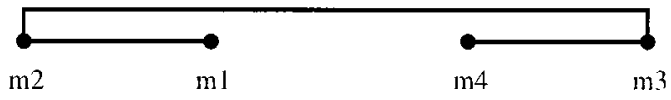

Fig. 2. Path of minimal length.

Take $\ell \in \mathbb{P}^{d}$ together with its orientation. A path of minimal length through the points $\pi_{\ell}\left(S_{n}\right)$ which connects $m_{1}$ with $m_{4}$ (see Fig. 2) consists of

$$
\operatorname{conv}\left\{m_{1}, m_{2}\right\} \cup \operatorname{conv}\left\{m_{2}, m_{3}\right\} \cup \operatorname{conv}\left\{m_{3}, m_{4}\right\} .
$$

That is, the points of $\ell$ between $m_{1}$ and $m_{2}$ are covered twice by a path of minimal length through the points $\pi_{\ell}\left(S_{n}\right)$, the points between $m_{1}$ and $m_{4}$ are covered once, and the points between $m_{3}$ and $m_{4}$ are covered twice again. If $\pi_{\ell}\left(P\left(S_{n}\right)\right)$ is not a path of minimal length through the points $\pi_{\ell}\left(S_{n}\right)$, then there has to exist an interval

$$
I=\left[\min _{\ell}\left\{\pi_{\ell}\left(p_{n}^{i}\right), \pi_{\ell}\left(p_{n}^{i+1}\right)\right\}, \max _{\ell}\left\{\pi_{\ell}\left(p_{n}^{i}\right), \pi_{\ell}\left(p_{n}^{i+1}\right)\right\}\right]
$$

on $\ell$ with $p_{n}^{i}, p_{n}^{i+1} \in S_{n}$, which is covered by $\pi_{\ell}\left(P\left(S_{n}\right)\right)$

1. $2+2 k$ times, $k \geq 1$, if

$$
m_{2} \leq \min _{\ell}\left\{\pi_{\ell}\left(p_{n}^{i}\right), \pi_{\ell}\left(p_{n}^{i+1}\right)\right\}<\max _{\ell}\left\{\pi_{\ell}\left(p_{n}^{i}\right), \pi_{\ell}\left(p_{n}^{i+1}\right)\right\} \leq m_{1}
$$

or

$$
m_{4} \leq \min _{\ell}\left\{\pi_{\ell}\left(p_{n}^{i}\right), \pi_{\ell}\left(p_{n}^{i+1}\right)\right\}<\max _{\ell}\left\{\pi_{\ell}\left(p_{n}^{i}\right), \pi_{\ell}\left(p_{n}^{i+1}\right)\right\} \leq m_{3},
$$

2. $1+2 k$ times, $k \geq 1$, if

$$
m_{1} \leq \min _{\ell}\left\{\pi_{\ell}\left(p_{n}^{i}\right), \pi_{\ell}\left(p_{n}^{i+1}\right)\right\}<\max _{\ell}\left\{\pi_{\ell}\left(p_{n}^{i}\right), \pi_{\ell}\left(p_{n}^{i+1}\right)\right\} \leq m_{4} .
$$

For all $p_{n}^{j} \in S_{n}, j \in\left\{2, \ldots,\left|S_{n}\right|-2\right\}$, we call the interval

$$
I_{j}=\left[\min _{\ell}\left\{\pi_{\ell}\left(p_{n}^{j}\right), \pi_{\ell}\left(p_{n}^{j+1}\right)\right\}, \max _{\ell}\left\{\pi_{\ell}\left(p_{n}^{j}\right), \pi_{\ell}\left(p_{n}^{j+1}\right)\right\}\right]
$$

positive if $\pi_{\ell}\left(p_{n}^{j}\right)<\pi_{\ell}\left(p_{n}^{j+1}\right)$ in the order on $\ell$ and negative otherwise. The intervals $I_{j}$ which cover the interval $I$ must have alternating signs. That is, if $\pi_{\ell}\left(P\left(S_{n}\right)\right)$ is not a shortest path through the points $\pi_{\ell}\left(S_{n}\right)$ we find, using that $P\left(S_{n}\right)$ connects the $p_{n}^{j} \in S_{n}$ in their order along $\gamma$ and using the continuity of $\gamma$ and of the projection map $\pi_{\ell}$, three points $p_{n}, q_{n}, r_{n} \in B_{1 / n}(p)$ with $p_{n}<q_{n}<r_{n} \leq p$ in the order along $\gamma$, but

$$
\pi_{\ell}\left(q_{n}\right)<\pi_{\ell}\left(p_{n}\right)<\pi_{\ell}\left(r_{n}\right) \quad \text { or } \quad \pi_{\ell}\left(p_{n}\right)<\pi_{\ell}\left(r_{n}\right)<\pi_{\ell}\left(q_{n}\right) \quad \text { in the order on } \ell \text {; }
$$

or we find three points $p_{n}, q_{n}, r_{n} \in B_{1 / n}(p)$ with $p_{n}>q_{n}>r_{n} \geq p$ in the order along $\gamma$, but

$$
\pi_{\ell}\left(q_{n}\right)>\pi_{\ell}\left(p_{n}\right)>\pi_{\ell}\left(r_{n}\right) \text { or } \pi_{\ell}\left(p_{n}\right)>\pi_{\ell}\left(r_{n}\right)>\pi_{\ell}\left(q_{n}\right) \quad \text { in the order on } \ell .
$$

See Fig. 3 for an example. 


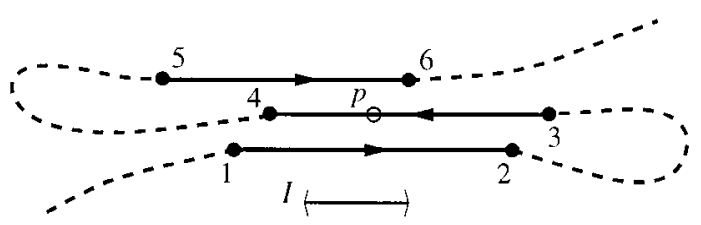

Fig. 3. Example: $4,5,6>p$ are not projected in the right order.

In general the points $p_{n}, q_{n}$, and $r_{n}$ need not be sample points. From Lemma 3.3 we can conclude that

$$
\lim _{n \rightarrow \infty} \mu_{d}\left(L_{n}\right)=0 .
$$

Second Step. Let $M_{n} \subset \mathbb{P}^{d}$ be the set of lines $\ell$ for which we have:

1. The order of $S_{n}$ along $\gamma$ and the order of $S_{n}$ induced by the order of $\pi_{\ell}\left(S_{n}\right)$ on $\ell$ coincide.

2. For all $\operatorname{conv}\left\{p^{i}, p^{i+1}\right\} \subset P\left(S_{n}\right)$ with $p^{i}, p^{i+1} \in S_{n}$ we have

$$
\left|\pi_{\ell}\left(p^{i+1}\right)-\pi_{\ell}\left(p^{i}\right)\right| \geq \frac{1}{2} \cos \left(\frac{\pi+\alpha}{4}\right)\left|p^{i+1}-p^{i}\right| .
$$

We show that there exist $c>0$ and $N^{\prime} \in \mathbb{N}$ such that for all $n \geq N^{\prime}$ we have

$$
\mu_{d}\left(M_{n}\right) \geq c .
$$

For the proof we construct a set of lines $C \subset \mathbb{P}^{d}$ with $\mu_{d}(C)>0$ and show that there exists $N^{\prime} \in \mathbb{N}$ such that $C \subset M_{n}$ for all $n \geq N^{\prime}$. The set $C$ is defined as follows: Let $\ell_{0}$ be the line, $\ell_{0} \subset \operatorname{span}\{1(p), \mathrm{r}(p)\}$ such that $\ell_{0}^{\perp} \subset \operatorname{span}\{1(p), \mathrm{r}(p)\}$ halves the angle $\angle(1(p),-\mathrm{r}(p))$ between the lines determined by $\mathrm{l}(p)$ and $\mathrm{r}(p)$. Now we define

$$
C=\left\{\ell \in \mathbb{P}^{d}: \angle\left(\ell, \ell_{0}\right) \leq \frac{1}{4}(\pi-\alpha)\right\} .
$$

By $\angle\left(\ell, \ell^{\prime}\right)$ for $\ell, \ell^{\prime} \in \mathbb{P}^{d}$ we denote the value of the minimum of the two angles determined by $\ell$ and $\ell^{\prime}$. The set $\bigcup_{\ell \in C}\{x \in \ell\} \subset \mathbb{R}^{d+1}$ is a double cone (see Fig. 4).

By construction $\pi_{\ell}(\mathrm{l}(p))$ and $\pi_{\ell}(\mathrm{r}(p))$ point in the same direction on every $\ell \in C$. Since $\alpha<\pi$ we have $\mu_{d}(C)>0$. It remains to check conditions 1 and 2 to prove that for sufficiently large $n$ we have $C \subset M_{n}$.

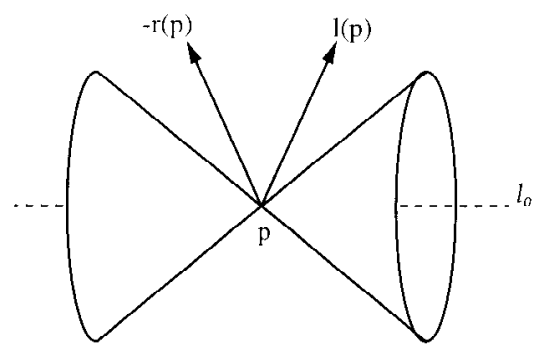

Fig. 4. The double cone C. 
1. Assume that for arbitrary large $n$ we find $\ell_{n} \in C$ such that the first condition is violated. Then we can find three points $p_{n}, q_{n}, r_{n} \in B_{1 / n}(p)$ with $p_{n}<q_{n}<r_{n} \leq p$ in the order along $\gamma$, but

$$
\pi_{\ell_{n}}\left(q_{n}\right)<\pi_{\ell_{n}}\left(p_{n}\right)<\pi_{\ell_{n}}\left(r_{n}\right) \quad \text { or } \quad \pi_{\ell_{n}}\left(p_{n}\right)<\pi_{\ell_{n}}\left(r_{n}\right)<\pi_{\ell_{n}}\left(q_{n}\right)
$$

in the order on $\ell_{n}$; or we find three points $p_{n}, q_{n}, r_{n} \in B_{1 / n}(p)$ with $p_{n}>q_{n}>r_{n} \geq p$ in the order along $\gamma$, but

$$
\pi_{\ell_{n}}\left(q_{n}\right)>\pi_{\ell_{n}}\left(p_{n}\right)>\pi_{\ell_{n}}\left(r_{n}\right) \quad \text { or } \quad \pi_{\ell_{n}}\left(p_{n}\right)>\pi_{\ell_{n}}\left(r_{n}\right)>\pi_{\ell_{n}}\left(q_{n}\right)
$$

in the order on $\ell_{n}$. By Lemma 3.1 the limit of every convergent subsequence of $\left(\ell_{n}\right)$ has to be perpendicular to either $1(p)$ or $\mathrm{r}(p)$. Since $C$ is compact we find $\ell \in C$ as the limit of a convergent subsequence of $\left(\ell_{n}\right)$ which is perpendicular to either $1(p)$ or $\mathrm{r}(p)$. On the other hand we find for all $\ell \in C$ using the triangle inequality for spherical triangles,

$$
\begin{aligned}
\angle(\ell, 1(p)) & \leq \angle\left(\ell, \ell_{0}\right)+\angle\left(\ell_{0}, 1(p)\right) \\
& <\frac{1}{4}(\pi-\alpha)+\left(\frac{\pi}{2}-\frac{\pi-\alpha}{2}\right) \\
& =\frac{\pi+\alpha}{4}<\frac{\pi}{2}
\end{aligned}
$$

and analogously

$$
\angle(\ell, \mathrm{r}(p))<\frac{\pi}{2}
$$

That is, every $\ell \in C$ is neither perpendicular to $1(p)$ nor to $\mathrm{r}(p)$. So we have a contradiction. That means, for all sufficiently large $n$ the first condition cannot be violated.

2. From the s-regularity of $\gamma$ and the continuity of $\angle$ we have, for $p_{n}^{i}, p_{n}^{i+1} \in S_{n}$ with $p_{n}^{i}<p_{n}^{i+1} \leq p$ and all $\ell \in C$,

$$
\begin{aligned}
\lim _{n \rightarrow \infty} \angle\left(\ell, \operatorname{conv}\left\{p_{n}^{i}, p_{n}^{i+1}\right\}\right) & =\angle\left(\ell, \lim _{n \rightarrow \infty} \operatorname{conv}\left\{p_{n}^{i}, p_{n}^{i+1}\right\}\right) \\
& =\angle(\ell, 1(p)) \leq \frac{\pi+\alpha}{4} .
\end{aligned}
$$

That is, for all sufficiently large $n$ and all $\ell \in C$ we have

$$
\left|\pi_{\ell}\left(p^{i}\right)-\pi_{\ell}\left(p^{i+1}\right)\right| \geq \frac{1}{2} \cos \left(\frac{\pi+\alpha}{4}\right)\left|p^{i}-p^{i+1}\right| .
$$

For $p_{n}^{i}, p_{n}^{i+1} \in S_{n}$ with $p \leq p_{n}^{i}<p_{n}^{i+1}$ we get the same result using $\mathrm{r}(p)$ instead of $1(p)$ in the triangle inequality for spherical triangles. It remains to consider the case that $p_{n}^{i}<p<p_{n}^{i+1}$. We choose $\eta>0$ such that

$$
\cos \left(\frac{\pi+\alpha}{4}+\eta\right)>\frac{1}{2} \cos \left(\frac{\pi+\alpha}{4}\right)
$$

and show that, for all $\ell \in C$ and all sufficiently large $n$,

$$
\angle\left(\ell, \operatorname{conv}\left\{p_{n}^{i}, p_{n}^{i+1}\right\}\right) \leq \frac{\pi+\alpha}{4}+\eta .
$$


To do so let $\pi$ be the projection on $\operatorname{span}\{1(p), \mathrm{r}(p)\}$. The s-regularity of $\gamma$ implies

$$
\lim \sup \left(\angle\left(\ell_{0}, \pi\left(\operatorname{conv}\left\{p_{n}^{i}, p_{n}^{i+1}\right\}\right)\right)\right) \leq \angle\left(\ell_{0}, 1(p)\right)=\angle\left(\ell_{0}, \mathrm{r}(p)\right) .
$$

We define the distance between two two-dimensional subspaces of $\mathbb{R}^{d+1}$ by the smaller of the two dihedral angles defined by them. With this metric the span seen as a mapping becomes continuous. From the continuity of $\angle$ and the continuity of span we get

$$
\begin{aligned}
0 & =\lim _{n \rightarrow \infty} \angle\left(\operatorname{span}\left\{\frac{p_{n}^{i}-p}{\left|p_{n}^{i}-p\right|}, \frac{p-p_{n}^{i+1}}{\left|p-p_{n}^{i+1}\right|}\right\}, \operatorname{conv}\left\{p_{n}^{i}, p_{n}^{i+1}\right\}\right) \\
& =\angle\left(\lim _{n \rightarrow \infty} \operatorname{span}\left\{\frac{p_{n}^{i}-p}{\left|p_{n}^{i}-p\right|}, \frac{p-p_{n}^{i+1}}{\left|p-p_{n}^{i+1}\right|}\right\}, \lim _{n \rightarrow \infty} \operatorname{conv}\left\{p_{n}^{i}, p_{n}^{i+1}\right\}\right) \\
& =\angle\left(\operatorname{span}\{1(p), \mathrm{r}(p)\}, \lim _{n \rightarrow \infty} \operatorname{conv}\left\{p_{n}^{i}, p_{n}^{i+1}\right\}\right) \\
& =\lim _{n \rightarrow \infty} \angle\left(\operatorname{span}\{1(p), \mathrm{r}(p)\}, \operatorname{conv}\left\{p_{n}^{i}, p_{n}^{i+1}\right\}\right) .
\end{aligned}
$$

That is, for all sufficiently large $n$ we have

$$
\angle\left(\pi\left(\operatorname{conv}\left\{p_{n}^{i}, p_{n}^{i+1}\right\}\right), \operatorname{conv}\left\{p_{n}^{i}, p_{n}^{i+1}\right\}\right) \leq \eta .
$$

Finally we get, for all sufficiently large $n$ using the inequalities (2), (3) and the triangle inequality for spherical triangles,

$$
\begin{aligned}
\angle\left(\ell, \operatorname{conv}\left\{p_{n}^{i}, p_{n}^{i+1}\right\}\right) \leq & \angle\left(\ell, \ell_{0}\right)+\angle\left(\ell_{0}, \operatorname{conv}\left\{p_{n}^{i}, p_{n}^{i+1}\right\}\right) \\
\leq & \angle\left(\ell, \ell_{0}\right)+\angle\left(\ell_{0}, \pi\left(\operatorname{conv}\left\{p_{n}^{i}, p_{n}^{i+1}\right\}\right)\right) \\
& +\angle\left(\pi\left(\operatorname{conv}\left\{p_{n}^{i}, p_{n}^{i+1}\right\}\right), \operatorname{conv}\left\{p_{n}^{i}, p_{n}^{i+1}\right\}\right) \\
\leq & \angle\left(\ell, \ell_{0}\right)+\angle\left(\ell_{0}, 1(p)\right)+\eta \\
\leq & \frac{\pi-\alpha}{4}+\frac{\alpha}{2}+\eta \\
= & \frac{\pi+\alpha}{4}+\eta .
\end{aligned}
$$

Hence for all sufficiently large $n$, all $\ell \in C$, and all $p_{n}^{i}, p_{n}^{i+1} \in S_{n}$ we have

$$
\left|\pi_{\ell}\left(p^{i}\right)-\pi_{\ell}\left(p^{i+1}\right)\right| \geq \frac{1}{2} \cos \left(\frac{\pi+\alpha}{4}\right)\left|p^{i}-p^{i+1}\right| .
$$

That is, there exists $N^{\prime} \in \mathbb{N}$ such that for all $n \geq N^{\prime}$ we have $C \subset M_{n}$. Hence

$$
\mu_{d}\left(M_{n}\right) \geq \mu_{d}(C)>0
$$

Third Step. In the first two steps we considered the measures of the subspaces $L_{n}$ and $M_{n}$ of $\mathbb{P}^{d}$, the space on which we want to integrate. In this step we compare integrands. 

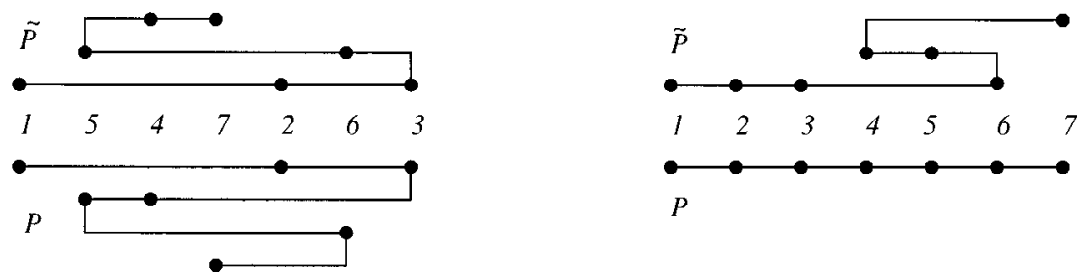

Fig. 5. Example: Projections on $\ell \in L_{n}$ (left) and on $\ell \in M_{n}$ (right).

Let $\tilde{P}\left(S_{n}\right)$ be a path different from $P\left(S_{n}\right)$ through the points $S_{n}$, which connects $p_{n}^{1}$ with $p_{n}^{\left|S_{n}\right|}$. We define the $\operatorname{rank} \operatorname{rk}\left(p_{n}^{i}, \tilde{P}\left(S_{n}\right)\right)$ of a sample point $p_{n}^{i} \in S_{n}$ in the path $\tilde{P}\left(S_{n}\right)$ as the number of sample points the path $\tilde{P}\left(S_{n}\right)$ meets on its way from $p_{n}^{1}$ to $p_{n}^{\left|S_{n}\right|}$ before it meets $p_{n}^{i}$.

Let $I_{n}$ be the following set of indices:

$$
P_{n}=\left\{i \in\left\{2, \ldots,\left|S_{n}\right|-2\right\}: \operatorname{rk}\left(p_{n}^{i}, \tilde{P}\left(S_{n}\right)\right)>\operatorname{rk}\left(p_{n}^{i+1}, \tilde{P}\left(S_{n}\right)\right)\right\} .
$$

If we compare the length of the projections $\pi_{\ell}\left(P\left(S_{n}\right)\right)$ and $\pi_{\ell}\left(\tilde{P}\left(S_{n}\right)\right)$ on $\ell \in L_{n}$ (see Fig. 5) we find

$$
L\left(\pi_{\ell}\left(P\left(S_{n}\right)\right)\right)-L\left(\pi_{\ell}\left(\tilde{P}\left(S_{n}\right)\right)\right) \leq 2 \sum_{i \in I_{n}}\left|\pi_{\ell}\left(p_{n}^{i}\right)-\pi_{\ell}\left(p_{n}^{i+1}\right)\right| .
$$

On the other hand we find, for the length of projections on $\ell \in M_{n}$ (see Fig. 5),

$$
L\left(\pi_{\ell}\left(\tilde{P}\left(S_{n}\right)\right)\right)-L\left(\pi_{\ell}\left(P\left(S_{n}\right)\right)\right) \geq 2 \sum_{i \in I_{n}}\left|\pi_{\ell}\left(p_{n}^{i}\right)-\pi_{\ell}\left(p_{n}^{i+1}\right)\right| .
$$

Furthermore, we have, for all $p_{n}^{i} \in S_{n}, i \in\left\{1, \ldots,\left|S_{n}\right|-1\right\}$ and all $\ell \in M_{n}$,

$$
\left|\pi_{\ell}\left(p^{i+1}\right)-\pi_{\ell}\left(p^{i+1}\right)\right| \geq \frac{1}{2} \cos \left(\frac{\pi+\alpha}{4}\right)\left|p^{i+1}-p^{i}\right| .
$$

From this inequality we get another inequality which is valid for all $\ell \in M_{n}$ and all $\ell^{\prime} \in L_{n}$,

$$
\sum_{i \in I_{n}}\left|\pi_{\ell}\left(p_{n}^{i}\right)-\pi_{\ell}\left(p_{n}^{i+1}\right)\right| \geq \frac{1}{2} \cos \left(\frac{\pi+\alpha}{4}\right) \sum_{i \in I_{n}}\left|\pi_{\ell^{\prime}}\left(p_{n}^{i}\right)-\pi_{\ell^{\prime}}\left(p_{n}^{i+1}\right)\right|
$$

which implies, together with (4) and (5),

$L\left(\pi_{\ell}\left(\tilde{P}\left(S_{n}\right)\right)\right)-L\left(\pi_{\ell}\left(P\left(S_{n}\right)\right)\right) \geq \frac{1}{2} \cos \left(\frac{\pi+\alpha}{4}\right)\left(L\left(\pi_{\ell^{\prime}}\left(P\left(S_{n}\right)\right)\right)-L\left(\pi_{\ell^{\prime}}\left(\tilde{P}\left(S_{n}\right)\right)\right)\right)$.

Since this inequality is valid for all $\ell \in M_{n}$ and all $\ell^{\prime} \in L_{n}$ we get for the increase of length on $M_{n}$

$$
\begin{aligned}
& \int_{M_{n}}\left(L\left(\pi_{\ell}\left(\tilde{P}\left(S_{n}\right)\right)\right)-L\left(\pi_{\ell}\left(P\left(S_{n}\right)\right)\right)\right) \mathrm{d} \mu_{d}(\ell) \\
& \quad \geq \mu_{d}\left(M_{n}\right) \frac{1}{2} \cos \left(\frac{\pi+\alpha}{4}\right) \sup _{\ell \in L_{n}}\left(L\left(\pi_{\ell}\left(P\left(S_{n}\right)\right)\right)-L\left(\pi_{\ell}\left(\tilde{P}\left(S_{n}\right)\right)\right)\right)
\end{aligned}
$$


and for the decrease of length on $L_{n}$

$$
\begin{aligned}
& \int_{L_{n}}\left(L\left(\pi_{\ell}\left(P\left(S_{n}\right)\right)\right)-L\left(\pi_{\ell}\left(\tilde{P}\left(S_{n}\right)\right)\right)\right) \mathrm{d} \mu_{d}(\ell) \\
& \quad \leq \mu_{d}\left(L_{n}\right) \sup _{\ell \in L_{n}}\left(L\left(\pi_{\ell}\left(P\left(S_{n}\right)\right)\right)-L\left(\pi_{\ell}\left(\tilde{P}\left(S_{n}\right)\right)\right)\right) .
\end{aligned}
$$

Because of $\lim _{n \rightarrow \infty} \mu_{d}\left(L_{n}\right)=0$ there exists $N \geq N^{\prime}$ such for all $n \geq N$ we have

$$
\mu_{d}\left(L_{n}\right)<\mu_{d}(C) \frac{1}{2} \cos \left(\frac{\pi+\alpha}{4}\right) \leq \mu_{d}\left(M_{n}\right) \frac{1}{2} \cos \left(\frac{\pi+\alpha}{4}\right) .
$$

Using Theorem 2.2 we find that for all $n \geq N$ there is no shortcut possible and that $P\left(S_{n}\right)$ is the unique path of minimal length through the points $S_{n}$ with fixed start- and endpoints, because the polygon connecting the points in the order induced by $\gamma$ has a shorter projection on all $\ell \in C$ than every other polygon through the points $S_{n}$ with fixed start- and endpoints.

\section{Menger's Theorem}

We need Menger's theorem and some corollaries to achieve the transition from the local results of the last section to the global.

Menger's elementary proof of his theorem is only valid for simple open curves. Here we give a new proof that also holds for simple closed curves. For this proof, which in contrast to the original proof of Menger is restricted to curves in Euclidean spaces, we need the following lemma.

Lemma 4.1. Let $\gamma$ be a simple curve and let $\left(S_{n}\right)$ be a sequence of samples of $\gamma$ with $\lim _{n \rightarrow \infty} \varepsilon\left(S_{n}\right)=0$ and $\lim \sup L\left(\operatorname{TSP}\left(S_{n}\right)\right)<\infty$. Let

$$
\gamma_{n}:\left[0, L\left(T S P\left(S_{n}\right)\right)\right] \rightarrow \mathbb{R}^{d}
$$

be the parameterization by length of $\operatorname{TSP}\left(S_{n}\right)$. Then the sequence $\left(\gamma_{n}\right)$ has a Fréchet convergent subsequence.

Proof. By turning to an appropriate subsequence we can assume that

$$
L\left(T S P\left(S_{n}\right)\right)<2 \lim \sup L\left(T S P\left(S_{n}\right)\right)=: c<\infty
$$

for all $n \in \mathbb{N}$. To prove the lemma it is sufficient to check that the premises of the theorem of Ascoli [9] are fulfilled.

1. The sequence of reduced parameterizations

$$
\gamma_{n}^{\prime}:[0,1] \rightarrow \mathbb{R}^{d}, t \mapsto \gamma_{n}\left(t L\left(T S P\left(S_{n}\right)\right)\right)
$$


is equicontinuous, because

$$
\begin{aligned}
\left|\gamma_{n}^{\prime}\left(t^{\prime}\right)-\gamma_{n}^{\prime}(t)\right| & \leq\left(t^{\prime}-t\right) L\left(\gamma_{n}^{\prime}\right) \\
& =\left(t^{\prime}-t\right) L\left(\operatorname{TSP}\left(S_{n}\right)\right) \\
& \leq c\left(t^{\prime}-t\right)
\end{aligned}
$$

for all $0 \leq t<t^{\prime} \leq 1$ and all $n \in \mathbb{N}$.

2. We have $\sup _{n \in \mathbb{N}} \sup \left\{\left|\gamma_{n}^{\prime}(t)\right|: t \in[0,1]\right\}<\infty$, because $\gamma$ is compact.

Now the theorem of Ascoli states that the sequence $\left(\gamma_{n}^{\prime}\right)$ has a convergent subsequence $\left(\gamma_{m}^{\prime}\right)$ which converges to a continuous function

$$
\gamma^{\prime}:[0,1] \rightarrow \mathbb{R}^{d}
$$

Note that the condition $\lim \sup L\left(\operatorname{TSP}\left(S_{n}\right)\right)<\infty$ is always fulfilled if $\gamma$ has finite length. Now we are prepared to prove Menger's theorem. In this proof we make use of the one-dimensional Hausdorff measure $\mathfrak{H}^{1}$, see [8] for the definition and its relation to the geometry of curves.

Theorem 4.1. Every simple curve $\gamma$ satisfies

$$
L(\gamma)=\sup \{L(T S P(S)): S \text { is a sample of } \gamma\},
$$

where $\operatorname{TSP}(S)$ denotes a shortest path through the sample points $S$ if $\gamma$ is an open curve and a shortest tour otherwise.

Proof. Assume the contrary. That is, we can assume that

$$
\sup \{L(T S P(S)): S \text { is a sample of } \gamma\}<L(\gamma),
$$

because $\operatorname{TSP}(S) \leq P(S) \leq L(\gamma)$ for every sample $S$ of $\gamma$. Take any sequence $\left(S_{n}\right)$ of samples from $\gamma$ with $\lim _{n \rightarrow \infty} \varepsilon\left(S_{n}\right)=0$. By our assumption we have

$$
\lim \sup L\left(T S P\left(S_{n}\right)\right)<L(\gamma) .
$$

Let $\gamma_{n}:[0,1] \rightarrow \mathbb{R}^{d}$ be the reduced parameterization of $\operatorname{TSP}\left(S_{n}\right)$. From Lemma 4.1 we know that there exists a Fréchet convergent subsequence $\left(\gamma_{m}\right)$ of $\left(\gamma_{n}\right)$. Let the continuous function $\gamma^{\prime}:[0,1] \rightarrow \mathbb{R}^{d}$ be the limit of $\left(\gamma_{m}\right)$. We have, for the convergent subsequence,

$$
\lim \sup L\left(T S P\left(S_{m}\right)\right) \leq \lim \sup L\left(T S P\left(S_{n}\right)\right)<L(\gamma) .
$$

Next we show that $\gamma \subset \gamma^{\prime}([0,1])$ : Take $p \in \gamma$. Since $\lim _{n \rightarrow \infty} \varepsilon\left(S_{n}\right)=0$, one finds a sequence $\left(p_{n}\right)$ with $p_{n} \in S_{n}$ which converges to $p$. Of course the subsequence $\left(p_{m}\right)$ with 
$p_{m} \in \gamma_{m}$ also converges to $p$. For all $k \in \mathbb{N}$ there exists $m(k) \in \mathbb{N}$ and $p_{m(k)}^{\prime} \in \gamma^{\prime}([0,1])$ such that

$$
\left|p_{m(k)}^{\prime}-p_{m(k)}\right|<\frac{1}{2 k} \quad \text { and } \quad\left|p_{m(k)}-p\right|<\frac{1}{2 k} .
$$

Hence by the triangle inequality we have $\left|p_{m(k)}^{\prime}-p\right|<1 / k$. From the compactness of $\gamma^{\prime}([0,1])$ we get $p \in \gamma^{\prime}([0,1])$.

Both $\gamma$ and $\gamma^{\prime}([0,1])$ are as compact sets $\mathfrak{H}^{1}$ measurable. Using the properties of the one-dimensional Hausdorff measure $\mathfrak{H}^{1}$, see [8], and our assumption we find

$$
\begin{aligned}
L(\gamma) & =\mathfrak{H}^{1}(\gamma) \\
& \leq \mathfrak{H}^{1}\left(\gamma^{\prime}([0,1])\right) \\
& \leq \liminf \mathfrak{H}^{1}\left(\operatorname{TSP}\left(S_{m}\right)\right) \\
& =\liminf \operatorname{L}\left(\operatorname{TSP}\left(S_{m}\right)\right) \\
& \leq \lim \sup \operatorname{L}\left(\operatorname{TSP}\left(S_{m}\right)\right) \\
& <L(\gamma) .
\end{aligned}
$$

That is a contradiction.

The following corollary states that the length of the traveling salesman path (tour) through a sequence of sample points converges to the length of the curve when the density of the sample goes to infinity. To avoid confusion we remark here that in the following $\pi$ and $\pi_{n}$ always denote permutations and no longer projections.

Corollary 4.1. Let $\gamma$ be a curve and let $\left(S_{n}\right)$ be a sequence of samples of $\gamma$, with $\lim _{n \rightarrow \infty} \varepsilon\left(S_{n}\right)=0$. Then

$$
L(\gamma)=\lim _{n \rightarrow \infty} L\left(T S P\left(S_{n}\right)\right),
$$

where $\operatorname{TSP}\left(S_{n}\right)$ denotes a shortest path through the sample points $S_{n}$ if $\gamma$ is an open curve and a shortest tour otherwise.

Proof. Assume $L(\gamma)<\infty$. For a given $\eta>0$ consider three sets:

1. From Menger's theorem we know that there exists a sample

$$
S=\left\{q^{1}, \ldots, q^{|S|}\right\} \quad \text { with } L(T S P(S))>L(\gamma)-\frac{\eta}{2} .
$$

2. Let $S^{\prime}=\left\{p^{1}, \ldots, p^{\left|S^{\prime}\right|}\right\} \in\left\{S_{n}: n \in \mathbb{N}\right\}$ be such that $\varepsilon\left(S^{\prime}\right)<\eta / 4|S|$.

3. Let $S^{\prime \prime}=\left\{r^{1}, \ldots, r^{|S|}\right\} \subset S^{\prime}$ be a multiset with $\left|r^{i}-q^{i}\right| \leq \varepsilon\left(S^{\prime}\right)$ for all $i=$ $1, \ldots,|S|$.

In the following we make use of the conventions for $|S|+1$ and $\left|S^{\prime}\right|+1$ introduced in Definition 2.2. Let $\pi$ be a permutation of $\{1, \ldots,|S|\}$ such that

$$
\sum_{i=1}^{|S|}\left|q^{\pi(i+1)}-q^{\pi(i)}\right|=L(\operatorname{TSP}(S)),
$$


let $\pi^{\prime}$ be a permutation of $\left\{1, \ldots,\left|S^{\prime}\right|\right\}$ such that

$$
\sum_{i=1}^{\left|S^{\prime}\right|}\left|p^{\pi^{\prime}(i+1)}-p^{\pi^{\prime}(i)}\right|=\operatorname{L}\left(\operatorname{TSP}\left(S^{\prime}\right)\right),
$$

and let $\pi^{\prime \prime}$ be a permutation of $\{1, \ldots,|S|\}$ such that

$$
\sum_{i=1}^{|S|}\left|r^{\pi^{\prime \prime}(i+1)}-r^{\pi^{\prime \prime}(i)}\right| \text { is minimal. }
$$

Then it follows that:

1.

$$
\sum_{i=1}^{|S|}\left|r^{\pi^{\prime \prime}(i+1)}-r^{\pi^{\prime \prime}(i)}\right| \leq \sum_{i=1}^{\left|S^{\prime}\right|}\left|p^{\pi^{\prime}(i+1)}-p^{\pi^{\prime}(i)}\right|
$$

by construction.

2.

$$
\sum_{i=1}^{|S|}\left|q^{\pi(i+1)}-q^{\pi(i)}\right| \leq \sum_{i=1}^{|S|}\left|q^{\pi^{\prime \prime}(i+1)}-q^{\pi^{\prime \prime}(i)}\right|
$$

by the definition of $\pi$.

3. From $\left|r^{\pi^{\prime \prime}(j)}-q^{\pi^{\prime \prime}(j)}\right|<\varepsilon\left(S^{\prime}\right)$ for all $j=1, \ldots,|S|$ and the triangle inequality it follows that

$$
|| r^{\pi^{\prime \prime}(i+1)}-r^{\pi^{\prime \prime}(i)}|-| q^{\pi^{\prime \prime}(i+1)}-q^{\pi^{\prime \prime}(i)}||<2 \varepsilon\left(S^{\prime}\right) .
$$

Combining these inequalities leads to

$$
\begin{aligned}
L\left(T S P\left(S^{\prime}\right)\right) & =\sum_{i=1}^{\left|S^{\prime}\right|}\left|p^{\pi^{\prime}(i+1)}-p^{\pi^{\prime}(i)}\right| \\
& \geq \sum_{i=1}^{|S|}\left|r^{\pi^{\prime \prime}(i+1)}-r^{\pi^{\prime \prime}(i)}\right| \\
& \geq \sum_{i=1}^{|S|}\left|q^{\pi^{\prime \prime}(i+1)}-q^{\pi^{\prime \prime}(i)}\right|-2|S| \varepsilon\left(S^{\prime}\right) \\
& \geq \sum_{i=1}^{|S|}\left|q^{\pi(i+1)}-q^{\pi(i)}\right|-2|S| \varepsilon\left(S^{\prime}\right) \\
& =L(T S P(S))-2|S| \varepsilon\left(S^{\prime}\right) \\
& >L(T S P(S))-\frac{\eta}{2} \\
& >L(\gamma)-\eta .
\end{aligned}
$$

Since $\eta$ can be arbitrary small, we are done. The proof in the case $L(\gamma)=\infty$ is quite similar. 
The next lemma states that the maximal length of a segment in a traveling salesman path (tour) of a sequence of sample points from a curve of finite length tends to zero as the density of the sample goes to infinity. The proof again makes use of the one-dimensional Hausdorff measure $\mathfrak{H}^{1}$.

Lemma 4.2. Assume that the curve $\gamma$ has finite length and let $\left(S_{n}\right)$ be a sequence of samples of $\gamma$ with $\lim _{n \rightarrow \infty} \varepsilon\left(S_{n}\right)=0$. For all $n \in \mathbb{N}$ let $\pi_{n}$ be the permutation of $\left\{1, \ldots,\left|S_{n}\right|\right\}$ induced by $\operatorname{TSP}\left(S_{n}\right)$. Then

$$
\lim _{n \rightarrow \infty} \max \left\{\left|p^{\pi_{n}(i+1)}-p^{\pi_{n}(i)}\right|: i=1, \ldots,\left|S_{n}\right|\right\}=0
$$

where $\pi_{n}\left(\left|S_{n}+1\right|\right)=\pi_{n}\left(\left|S_{n}\right|\right)$ if $\gamma$ is an open curve and $\pi_{n}\left(\left|S_{n}+1\right|\right)=\pi_{n}(1)$ if $\gamma$ is closed.

Proof. Assume the contrary. That is, by choosing an appropriate subsequence one can assume that there exists $c>0$ such that for every $n \in \mathbb{N}$ it holds that

$$
\max \left\{\left|p^{\pi_{n}(i+1)}-p^{\pi_{n}(i)}\right|: i=1, \ldots,\left|S_{n}\right|\right\}>c .
$$

Let $p_{n}=p^{\pi_{n}(i)} \in S_{n}$ and $q_{n}=p^{\pi_{n}(i+1)} \in S_{n}$ be such that $\left|p_{n}-q_{n}\right|>c$. By the compactness of $\gamma$ we can turn to an appropriate subsequence such that $\left(p_{n}\right)$ converges to $p \in \gamma$ and $\left(q_{n}\right)$ converges to $q \in \gamma$. Of course it holds that $|p-q| \geq c$. Remove the interior of $\operatorname{conv}\left\{p_{n}, q_{n}\right\}$ from $\operatorname{TSP}\left(S_{n}\right)$. We consider two cases:

First, if $\gamma$ is an open curve, then $\operatorname{TSP}\left(S_{n}\right)$ decomposes into two paths $P_{n}^{1}$ which connects $p^{\pi_{n}(1)}$ with $p^{\pi_{n}(i)}$ and $P_{n}^{2}$ which connects $p^{\pi_{n}(i+1)}$ with $p^{\pi_{n}\left(\left|S_{n}\right|\right)}$. Let $P_{n}$ denote $P_{n}^{1} \cup P_{n}^{2}$ and let $\gamma_{n}^{j}:[0,1] \rightarrow \mathbb{R}^{d}, j=1,2$, be the reduced parameterization of $P_{n}^{j}$. As in Lemma 4.1 one can use the theorem of Ascoli to show that $\left(\gamma_{n}^{j}\right)$ has a Fréchet convergent subsequence. That is, we can assume by considering a common subsequence that $\left(\gamma_{n}^{1}\right)$ and $\left(\gamma_{n}^{2}\right)$ converge to continuous functions $\gamma^{1}:[0,1] \rightarrow \mathbb{R}^{d}$ and $\gamma^{2}:[0,1] \rightarrow \mathbb{R}^{d}$. Let $\gamma^{\prime}$ be $\gamma^{1}([0,1]) \cup \gamma^{2}([0,1])$. Second, if $\gamma$ is a closed curve, then $\operatorname{TSP}\left(S_{n}\right)$ becomes after the removal of the interior of $\operatorname{conv}\left\{p_{n}, q_{n}\right\}$ a path denoted by $P_{n}$ which connects $p^{\pi_{n}(i)}$ with $p^{\pi_{n}(i+1)}$. Let $\gamma_{n}$ be the reduced parameterization of $P_{n}$. As in Lemma 4.1 one can show that $\left(\gamma_{n}\right)$ has a Fréchet convergent subsequence. That is, we can assume by taking an appropriate subsequence that $\left(\gamma_{n}\right)$ converges to a continuous function $\gamma^{\prime}:[0,1] \rightarrow \mathbb{R}^{d}$. We use the symbol $\gamma^{\prime}$ also to denote the set $\gamma^{\prime}([0,1])$.

Next we show that $\gamma \subset \gamma^{\prime}$ : Take $r \in \gamma$. Since $\lim _{n \rightarrow \infty} \varepsilon\left(S_{n}\right)=0$, one finds a sequence $\left(r_{n}\right)$ with $r_{n} \in S_{n}$ which converges to $r$. For all $k \in \mathbb{N}$ there exists $n(k) \in \mathbb{N}$ and $r_{n(k)}^{\prime} \in \gamma^{\prime}$ such that

$$
\left|r_{n(k)}^{\prime}-r_{n(k)}\right|<\frac{1}{2 k} \quad \text { and } \quad\left|r_{n(k)}-r\right|<\frac{1}{2 k}
$$

Hence by the triangle inequality $\left|r_{n(k)}^{\prime}-r\right|<1 / k$. From the compactness of $\gamma^{\prime}$ we get $r \in \gamma^{\prime}$. 
The sets $\gamma, \gamma^{\prime}, P_{n}$, and $\operatorname{conv}\left\{p_{n}, q_{n}\right\}$ are all as compact sets $\mathfrak{H}^{1}$ measurable. Using the properties of the one-dimensional Hausdorff measure $\mathfrak{H}^{1}$, see [8], we find

$$
\begin{aligned}
\mathfrak{H}^{1}\left(\gamma^{\prime}\right) & \geq \mathfrak{H}^{1}\left(\gamma \cup \operatorname{conv}\left\{p_{n}, q_{n}\right\}\right) \\
& =\mathfrak{H}^{1}(\gamma)+\mathfrak{H}^{1}(\operatorname{conv}\{p, q\}) \\
& >\mathfrak{H}^{1}(\gamma) \\
& =L(\gamma) \\
& \geq \sup L\left(P_{n}\right) \\
& =\sup \mathfrak{H}^{1}\left(P_{n}\right) \\
& \geq \mathfrak{H}^{1}\left(\gamma^{\prime}\right) .
\end{aligned}
$$

That is a contradiction.

\section{From Local to Global}

In this section we finally want to prove the promised theorem. That is, here we achieve the transition from the local version of the theorem to the global. To do so we need another definition.

Definition 5.1. Given a curve $\gamma$ and a sample $S$ from $\gamma$. We call $r \in S$ a return point, if $r$ is connected to $p, q \in S$ in a traveling salesman path (tour) of $S$ and $r \triangleleft p, q$ or $r \triangleright p, q$ in the order along $\gamma$. In the first case we call the return point positive and in the second case we call it negative. For open curves we also call the start- and endpoints of $\operatorname{TSP}(S)$ the return points.

For example in Fig. 1 points 2 and 3 are return points. In the traveling salesman path, point 2 is connected to points 3 and 4, which are both larger than point 2 in the order along the curve. Point 3 is connected to points 1 and 2, which are both smaller than point 3 in the order along the curve. Hence point 2 is a negative return point and point 3 is a positive return point.

First we prove the global result for closed curves. The proof is again subdivided into three steps.

Theorem 5.1. Let $\gamma$ be a closed curve. Assume

$$
\alpha=\sup \{\angle(1(q), \mathrm{r}(q)): q \in \gamma\}<\pi
$$

and let $\left(S_{n}\right)$ be a sequence of samples of $\gamma$ with $\lim _{n \rightarrow \infty} \varepsilon\left(S_{n}\right)=0$. Then there exists $N \in \mathbb{N}$ such that $\operatorname{TSP}\left(S_{n}\right)=P\left(S_{n}\right)$ for all $n \geq N$. Here $\operatorname{TSP}\left(S_{n}\right)$ is a shortest tour through the points $S_{n}$. Furthermore, $\operatorname{TSP}\left(S_{n}\right)$ is unique for all $n \geq N$.

Proof. The proof is done by contradiction. Assume without loss of generality that $\operatorname{TSP}\left(S_{n}\right) \neq P\left(S_{n}\right)$ for all $n \in \mathbb{N}$. 
First Step. We show that for large $n$ there exist at least four return points. First we show that there exists at least one return point for large $n$. Assume the contrary. That is, there does not exist a return point in $S_{n}$ for arbitrary large $n$. By turning to a subsequence one can assume without loss of generality that there does not exist a return point for all $n \in \mathbb{N}$. Since $\operatorname{TSP}\left(S_{n}\right) \neq P\left(S_{n}\right)$ there exists $p_{n}^{i} \in S_{n}$ which is not connected to $p_{n}^{i-1}$ in $\operatorname{TSP}\left(S_{n}\right)$. Cut $\operatorname{TSP}\left(S_{n}\right)$ in two polygonal arcs $P_{n}^{1}$, with startpoint $p_{n}^{i}$ and endpoint $p_{n}^{i-1}$, and $P_{n}^{2}$, with startpoint $p_{n}^{i-1}$ and endpoint $p_{n}^{i}$. By our assumption that there does not exist a return point in $S_{n}$ the sample points in both polygonal arcs are connected in their order along $\gamma$.

From Lemma 4.2 and Definition 2.4 of the Jordan length we can conclude that

$$
\lim _{n \rightarrow \infty} L\left(P_{n}^{1}\right), \lim _{n \rightarrow \infty} L\left(P_{n}^{2}\right)=L(\gamma)
$$

That is, $\lim _{n \rightarrow \infty} L\left(T S P\left(S_{n}\right)\right)=2 L(\gamma)$. Which is a contradiction. Hence there has to exist a return point for large $n$.

Observe that the signs, see Definition 5.1, of the return points in $S_{n}$ always sum to zero and that return points incident along $\operatorname{TSP}\left(S_{n}\right)$ always have different signs. So one can conclude that for sufficiently large $n$ there exist at least two return points. Now assume that we have only two return points $p_{n}$ and $q_{n}$ for arbitrary large $n$. Cut $\operatorname{TSP}\left(S_{n}\right)$ into two polygonal $\operatorname{arcs} P_{n}^{1}$ and $P_{n}^{2}$ that connect $p_{n}$ with $q_{n}$. The points along these arcs, running from $p_{n}$ to $q_{n}$, are ordered in the same way as they are ordered along $\gamma$. From Lemma 4.2 and Definition 2.4 of the Jordan length we can conclude that

$$
\lim _{n \rightarrow \infty} L\left(P_{n}^{1}\right), \lim _{n \rightarrow \infty} L\left(P_{n}^{2}\right)=L(\gamma) .
$$

That is, $\lim _{n \rightarrow \infty} L\left(\operatorname{TSP}\left(S_{n}\right)\right)=2 L(\gamma)$. Which is a contradiction to Corollary 4.1. Hence for large $n$ there are at least four return points in $S_{n}$.

Second Step. We show that for large $n$ there must exist two return points $r_{n}^{1} \triangleleft r_{n}^{2}$ neighborly along $\operatorname{TSP}\left(S_{n}\right)$, i.e., $r_{n}^{1}$ and $r_{n}^{2}$ are consequent return points along $\operatorname{TSP}\left(S_{n}\right)$, such that the other return points $\tilde{r}_{n}^{1}$ neighborly to $r_{n}^{1}$ and $\tilde{r}_{n}^{2}$ neighborly to $r_{n}^{2}$ along $\operatorname{TSP}\left(S_{n}\right)$ are not in between $r_{n}^{1}$ and $r_{n}^{2}$. That is, one cannot find the following situation:

$$
r_{n}^{1} \triangleleft \tilde{r}_{n}^{1} \triangleleft r_{n}^{2} \quad \text { or } \quad r_{n}^{1} \triangleleft \tilde{r}_{n}^{2} \triangleleft r_{n}^{2} .
$$

However, it is possible that $\tilde{r}_{n}^{1}=r_{n}^{2}$ and $\tilde{r}_{n}^{2}=r_{n}^{1}$.

Assume that for all neighborly return points one finds situation (6) then all return points have to accumulate in between two return points (see Fig. 6). That is impossible since $\operatorname{TSP}\left(S_{n}\right)$ is closed.

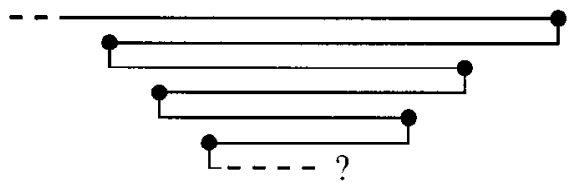

Fig. 6. Accumulating return points. 


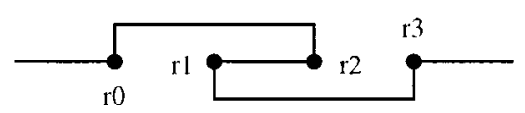

Fig. 7. Shortcut through return points.

Third Step. In this step the transition from the local version of the theorem to the global one is achieved. In this transition use is made of the return points $r_{n}^{1}$ and $r_{n}^{2}$ found in the second step. Choose the orientation of $\operatorname{TSP}\left(S_{n}\right)$ such that $r_{n}^{2} \triangleleft r_{n}^{1}$ along $\operatorname{TSP}\left(S_{n}\right)$. Let $r_{n}^{0} \in S_{n}$ be the last sample point one encounters running through $\operatorname{TSP}\left(S_{n}\right)$ with

$$
r_{n}^{0} \triangleleft r_{n}^{1} \text { along } \gamma \text { and } r_{n}^{0} \triangleleft r_{n}^{2} \text { along } \operatorname{TSP}\left(S_{n}\right)
$$

and let $r_{n}^{3} \in S_{n}$ be the first sample point one encounters running through $\operatorname{TSP}\left(S_{n}\right)$ with

$$
r_{n}^{2} \triangleleft r_{n}^{3} \text { along } \gamma \text { and } r_{n}^{1} \triangleleft r_{n}^{3} \text { along } \operatorname{TSP}\left(S_{n}\right) \text {. }
$$

That is, one finds the situation shown in Fig. 7.

By the compactness of $\gamma$ we can assume by turning to convergent subsequences that $\left(r_{n}^{0}\right),\left(r_{n}^{1}\right),\left(r_{n}^{2}\right)$, and $\left(r_{n}^{3}\right)$ converge to $r^{0}, r^{1}, r^{2}, r^{3} \in \gamma$. Let $s_{n} \in S_{n}$ be the successor of $r_{n}^{0}$ and let $p_{n} \in S_{n}$ be the predecessor of $r_{n}^{3}$ along $\operatorname{TSP}\left(S_{n}\right)$. By construction we have

$$
r_{n}^{0} \triangleleft r_{n}^{1} \triangleleft s_{n} \text { and } p_{n} \triangleleft r_{n}^{2} \triangleleft r_{n}^{3} \text { along } \gamma \text {. }
$$

From Lemma 4.2 we can conclude that

$$
\lim _{n \rightarrow \infty}\left|r_{n}^{0}-s_{n}\right|=\lim _{n \rightarrow \infty}\left|p_{n}-r_{n}^{3}\right|=0 .
$$

That is, $r^{0}=r^{1}$ and $r^{2}=r^{3}$. Now assume $r^{1} \triangleleft r^{2}$. Consider three sets of sample points

$$
\begin{aligned}
& M_{n}^{1}=\left\{p \in S_{n}: p \unlhd r_{n}^{2} \text { along } \operatorname{TSP}\left(S_{n}\right)\right\}, \\
& M_{n}^{2}=\left\{p \in S_{n}: r_{n}^{2} \unlhd p \unlhd r_{n}^{1} \text { along } \operatorname{TSP}\left(S_{n}\right)\right\}, \\
& M_{n}^{3}=\left\{p \in S_{n}: p \unlhd r_{n}^{1} \text { along } \operatorname{TSP}\left(S_{n}\right)\right\} .
\end{aligned}
$$

Using Corollary 4.1 and Theorem 4.1 one has

$$
\begin{aligned}
\lim _{n \rightarrow \infty} L & \left(\operatorname{TSP}\left(S_{n}\right)\right) \\
& \geq \lim _{n \rightarrow \infty}\left(L\left(\operatorname{TSP}\left(M_{n}^{1}\right)\right)+L\left(\operatorname{TSP}\left(M_{n}^{2}\right)\right)+L\left(\operatorname{TSP}\left(M_{n}^{3}\right)\right)\right) \\
& =\lim _{n \rightarrow \infty} L\left(\operatorname{TSP}\left(M_{n}^{1}\right)\right)+\lim _{n \rightarrow \infty} L\left(\operatorname{TSP}\left(M_{n}^{2}\right)\right)+\lim _{n \rightarrow \infty} L\left(\operatorname{TSP}\left(M_{n}^{3}\right)\right) \\
& =L\left(\left.\gamma\right|_{\left[0, \gamma^{-1}\left(r^{2}\right)\right]}\right)+L\left(\left.\gamma\right|_{\left[\gamma^{-1}\left(r^{1}\right), \gamma^{-1}\left(r^{2}\right)\right]}\right)+L\left(\left.\gamma\right|_{\left[\gamma^{-1}\left(r^{1}\right), 1\right]}\right) \\
& =L(\gamma)+2 L\left(\left.\gamma\right|_{\left[\gamma^{-1}\left(r^{1}\right), \gamma^{-1}\left(r^{2}\right)\right]}\right) \\
& >L(\gamma) .
\end{aligned}
$$

That is a contradiction to Corollary 4.1. Hence we have

$$
r^{0}=r^{1}=r^{2}=r^{3}=: r \in \gamma .
$$



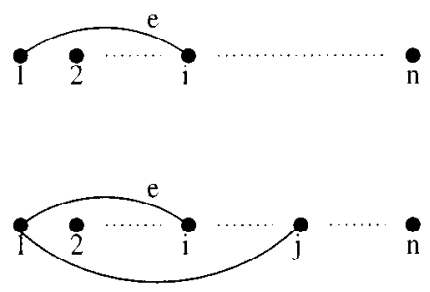

Fig. 8. Two cases in the induction.

By turning to an appropriate subsequence of $\left(S_{n}\right)$ we can assume without loss of generality that

$$
r_{n}^{0}, r_{n}^{1}, r_{n}^{2}, r_{n}^{3} \in S_{n} \cap B_{1 / n}(r) .
$$

That is a contradiction to Theorem 3.1, which is the local version of this theorem.

Next we want prove the global result for open curves. To do so we start with three lemmas.

Lemma 5.1. Let $S$ be a sample of an open curve $\gamma$, let $E(S)$ be the set of edges of $P(S)$, and let $E^{\prime}(S)$ be the set of edges of $P^{\prime}(S)$, where $P^{\prime}(S)$ is another polygonal path on $S$. Let $\pi$ be the permutation of $\{1, \ldots,|S|\}$ induced by $P^{\prime}(S)$. Then there exists a bijection $f: E^{\prime}(S) \rightarrow E(S)$ with

1. $f: \operatorname{conv}\left\{p^{\pi(i)}, p^{\pi(i+1)}\right\} \mapsto \operatorname{conv}\left\{p^{j}, p^{j+1}\right\}$ with $\pi(i) \leq j$ and $j+1 \leq \pi(i+1)$.

2. $\left.f\right|_{E(S) \cap E^{\prime}(S)}=\mathrm{id}$.

Proof. The proof is done by induction on the cardinality of $S$. If $|S|=2$ we must have $P(S)=P^{\prime}(S)$ and set $f=\mathrm{id}$. Assume the lemma is proven for $|S|<n$. Now assume that $|S|=n$. We distinguish two cases, which are depicted graphically in Fig. 8. The lines in this figure denote edges of $P_{\min }(S)$. In both cases map the edge $e$ to $\operatorname{conv}\left\{p^{1}, p^{2}\right\}$. Now remove $p^{1}$ and consider the induced polygon $\operatorname{TSP}\left(S-\left\{p^{1}\right\}\right)$ on the vertex set $\left\{p^{2}, \ldots, p^{n}\right\}$. In the second case add $\operatorname{conv}\left\{p^{i}, p^{j}\right\}$ to the induced polygon $\operatorname{TSP}\left(S-\left\{p^{1}\right\}\right)$. We are left with the problem of finding a suitable bijection on edge sets with $n-1$ elements. That is, we have reduced the problem to finding an appropriate bijection to the case $|S|=n-1$.

The next two lemmas are about regular curves. Nevertheless we can make use of them, since an open s-regular curve is regular in its endpoints.

Lemma 5.2. Let $\gamma$ be a regular curve. Then there exists an $\varepsilon>0$ such that

1. $\left|p^{i}-p^{i-1}\right|<\left|p^{i}-p^{k}\right|$ if $p^{k} \triangleleft p^{i-1}$,

2. $\left|p^{i}-p^{i+1}\right|<\left|p^{i}-p^{k}\right|$ if $p^{k} \triangleright p^{i+1}$

for all samples $S=\left\{p^{1}, \ldots, p^{|S|}\right\}$ with $\varepsilon(S)<\varepsilon$ and all $i \in\{1, \ldots,|S|\}$. 
Proof. Assume the contrary. Then there exists a sequence $\left(S_{n}\right)$ of samples with $\lim _{n \rightarrow \infty} \varepsilon\left(S_{n}\right)=0$ and $p_{n}^{i}, p_{n}^{k} \in S_{n}$ such that

$$
p^{k} \triangleleft p^{i-1} \text { and }\left|p_{n}^{i}-p_{n}^{i-1}\right| \geq\left|p_{n}^{i}-p_{n}^{k}\right|
$$

or

$$
p^{k} \triangleright p^{i+1} \text { and }\left|p_{n}^{i}-p_{n}^{i+1}\right| \geq\left|p_{n}^{i}-p_{n}^{k}\right|
$$

By choosing a subsequence one can always assume that for all $n \in \mathbb{N}$ one of the above possibilities holds. Without loss of generality assume that this is the first one. Since $\gamma$ is compact, one can also assume by choosing a subsequence that $\left(p_{n}^{i}\right)$ converges to $p \in \gamma$. From $\lim _{n \rightarrow \infty} \varepsilon\left(S_{n}\right)=0$ it follows that

$$
\lim _{n \rightarrow \infty}\left|p_{n}^{i}-p_{n}^{k}\right| \leq \lim _{n \rightarrow \infty}\left|p_{n}^{i}-p_{n}^{i-1}\right|=0
$$

Hence $\left(p_{n}^{i-1}\right)$ and $\left(p_{n}^{k}\right)$ also converge to $p$. Now look at the triangle with vertices $p_{n}^{i-1}, p_{n}^{i}$, and $p_{n}^{k}$. From the law of cosines together with $\left|p_{n}^{i}-p_{n}^{i-1}\right| \geq\left|p_{n}^{i}-p_{n}^{k}\right|$, it follows for the angle $\alpha_{n}$ at $p_{n}^{i-1}$ that

$$
\cos \left(\alpha_{n}\right)=-\frac{\left|p_{n}^{i}-p_{n}^{k}\right|^{2}-\left|p_{n}^{i}-p_{n}^{i-1}\right|^{2}-\left|p_{n}^{i-1}-p_{n}^{k}\right|^{2}}{2\left|p_{n}^{i}-p_{n}^{i-1}\right|\left|p_{n}^{i-1}-p_{n}^{k}\right|} \geq 0 .
$$

Thus $\alpha_{n}$ has to be smaller than or equal to $\pi / 2$, but that is a contradiction to Lemma 3.1. here.

The proof of the third lemma is similar to the proof of the second one, so we omit it

Lemma 5.3. Let $\gamma$ be a regular curve. Then there exists an $\varepsilon>0$ such that

1. $\left|p^{i}-p^{m}\right|<\left|p^{i}-p^{k}\right|$ for $p^{k} \triangleleft p^{m} \triangleleft p^{i}$, if $p^{k} \triangleleft p^{i-1}$ and $\left|p^{i}-p^{k}\right| \leq\left|p^{l}-p^{l+1}\right|$ for some $l \in\{1, \ldots,|S|\}$,

2. $\left|p^{i}-p^{m}\right|<\left|p^{i}-p^{k}\right|$ for $p^{i} \triangleleft p^{m}<\triangleleft p^{k}$, if $p^{k} \triangleright p^{i+1}$ and $\left|p^{i}-p^{k}\right| \leq\left|p^{l}-p^{l+1}\right|$ for some $l \in\{1, \ldots,|S|\}$

for all samples $S=\left\{p^{1}, \ldots, p^{|S|}\right\}$ with $\varepsilon(S)<\varepsilon$ and all $i \in\{1, \ldots,|S|\}$.

Now we are prepared to prove the global result for open curves.

Theorem 5.2. Let $\gamma$ be an open curve. Assume

$$
\alpha=\sup \{\angle(1(q), \mathrm{r}(q)): q \in \gamma\}<\pi
$$

and let $\left(S_{n}\right)$ be a sequence of samples of $\gamma$ with $\lim _{n \rightarrow \infty} \varepsilon\left(S_{n}\right)=0$. Then there exists $N \in \mathbb{N}$ such that $\operatorname{TSP}\left(S_{n}\right)=P\left(S_{n}\right)$ for all $n \geq N$. Here $\operatorname{TSP}\left(S_{n}\right)$ is a shortest path through the points $S_{n}$. Furthermore, $\operatorname{TSP}\left(S_{n}\right)$ is unique for all $n \geq N$. 
Proof. The proof is done by contradiction. Assume without loss of generality that $\operatorname{TSP}\left(S_{n}\right) \neq P\left(S_{n}\right)$ for all $n \in \mathbb{N}$.

First Step. We show that for large $n$ there exist at least four return points. Because $\operatorname{TSP}\left(S_{n}\right) \neq P\left(S_{n}\right)$ there have to exist at least three return points. If in $S_{n}$ there is only one return point besides the start- and endpoints of $\operatorname{TSP}\left(S_{n}\right)$, then this point is either $\min S_{n}$ or max $S_{n}$ along $\gamma$. Assume that there exists $N \in \mathbb{N}$ such that the number of return points in $S_{n}$ is three for all $n \geq N$. Without loss of generality we can assume that the only return point besides the endpoints of $\operatorname{TSP}\left(S_{n}\right)$ is $p_{n}^{1}=\min S_{n}$.

In $\operatorname{TSP}\left(S_{n}\right)$ we also have that $p_{n}^{1}$ is connected to $p_{n}^{2}$, because otherwise $S_{n}$ has more than three return points. Cut $\operatorname{TSP}\left(S_{n}\right)$ into two polygonal $\operatorname{arcs} P_{n}^{1}$ and $P_{n}^{2}$ with endpoint $p_{n}^{1}$. Since $S$ contains only three return points one of these arcs has as its second endpoint $p_{n}^{\left|S_{n}\right|}$. Assume without loss of generality that this arc is always $P_{n}^{1}$. From Lemma 4.2 and the definition of Jordan length we can conclude that $\lim _{n \rightarrow \infty} L\left(P_{n}^{1}\right)=L(\gamma)$. This implies

$$
\lim _{n \rightarrow \infty} L\left(P_{n}^{2}\right)=0 .
$$

Let $p_{n}^{i}, i \in\left\{3, \ldots,\left|S_{n}\right|\right\}$, be the second endpoint of $P_{n}^{2}$. We observe two things:

1. $\lim _{n \rightarrow \infty}\left|p_{n}^{1}-p_{n}^{i}\right|=0$, because $\left|p_{n}^{1}-p_{n}^{i}\right| \leq L\left(P_{n}^{2}\right)$ for large $n$.

2. $\operatorname{conv}\left\{p_{n}^{j}, p_{n}^{j+1}\right\} \subset \operatorname{TSP}\left(S_{n}\right)$ for all $i<j<\left|S_{n}\right|$ and large $n$. That is, the shortcuts take place on the first $i$ indices. Using that $\operatorname{TSP}\left(S_{n}\right) \neq P\left(S_{n}\right)$ and the statement and notions of Lemma 5.1, with $P^{\prime}(S)=T S P(S)$, we find that there exists

$$
\operatorname{conv}\left\{p_{n}^{\pi(j)}, p_{n}^{\pi(j+1)}\right\} \in E^{\prime}\left(S_{n}\right) \quad \text { and } \operatorname{conv}\left\{p_{n}^{k}, p_{n}^{k+1}\right\} \in E\left(S_{n}\right)
$$

with

$$
\begin{gathered}
\left|p_{n}^{\pi(j)}-p_{n}^{\pi(j+1)}\right| \leq\left|p_{n}^{k}-p_{n}^{k+1}\right|, \\
j, k<i, \quad \pi(j) \leq k, \quad k+1 \leq \pi(j+1), \quad \text { and } \quad \pi(j) \neq k \text { or } k+1 \neq \pi(j+1) .
\end{gathered}
$$

From $\lim _{n \rightarrow \infty} \varepsilon\left(S_{n}\right)=0$ we can conclude that $p_{n}^{1}$ converges to $\gamma(0)$. Since $\gamma(0)$ is a regular point of $\gamma$ we can apply here Lemmas 5.2 and 5.3. Now Lemma 5.3 tells us that there exists $n \in \mathbb{N}$ with:

1. $\left|p_{n}^{k}-p_{n}^{\pi(j+1)}\right| \leq\left|p_{n}^{\pi(j)}-p_{n}^{\pi(j+1)}\right|$ for all $n \geq N$. That means

$$
\left|p_{n}^{k}-p_{n}^{\pi(j+1)}\right|<\left|p_{n}^{k}-p_{n}^{k+1}\right| \quad \text { for all } n \geq N .
$$

2. $\left|p_{n}^{k+1}-p_{n}^{\pi(j)}\right| \leq\left|p_{n}^{\pi(j)}-p_{n}^{\pi(j+1)}\right|$ for all $n \geq N$. That means

$$
\left|p_{n}^{k+1}-p_{n}^{\pi(j)}\right| \leq\left|p_{n}^{k}-p_{n}^{k+1}\right| \quad \text { for all } n \geq N
$$

That is a contradiction to Lemma 5.2. Hence for large $n$ there are at least four return points in $S_{n}$.

Second Step. We show that for large $n$ there must exist two return points $r_{n}^{1} \triangleleft r_{n}^{2}$ incident along $\operatorname{TSP}\left(S_{n}\right)$ such that the other return points $\tilde{r}_{n}^{1}$ incident to $r_{n}^{1}$ and $\tilde{r}_{n}^{2}$ incident to $r_{n}^{2}$ 
along $\operatorname{TSP}\left(S_{n}\right)$ are not in between $r_{n}^{1}$ and $r_{n}^{2}$. That is, one cannot find the following situation:

$$
r_{n}^{1} \triangleleft \tilde{r}_{n}^{1} \triangleleft r_{n}^{2} \quad \text { or } \quad r_{n}^{1} \triangleleft \tilde{r}_{n}^{2} \triangleleft r_{n}^{2} .
$$

However, it is possible that $\tilde{r}_{n}^{1}=r_{n}^{2}$ and $\tilde{r}_{n}^{2}=r_{n}^{1}$.

The path $P_{n}^{1} \subset \operatorname{TSP}\left(S_{n}\right)$ connecting $p_{n}^{1}$ with $p_{n}^{\left|S_{n}\right|}$ either contains two return points, as we are looking for, or it does not contain any return point besides $p_{n}^{1}$ and $p_{n}^{\left|S_{n}\right|}$. So assume that $P_{n}^{1}$ does not contain any return point besides $p_{n}^{1}$ and $p_{n}^{\left|S_{n}\right|}$. Cut $\operatorname{TSP}\left(S_{n}\right)$ into three paths $P_{n}^{1}, P_{n}^{2}$ with endpoint $p_{n}^{1}$ and $P_{n}^{3}$ with endpoint $p_{n}^{\left|S_{n}\right|}$. At least one of the the paths $P_{n}^{2}, P_{n}^{3}$ cannot be empty since $S_{n}$ has at least four return points. From Lemma 4.2 and the definition of Jordan length we can conclude that $\lim _{n \rightarrow \infty} L\left(P_{n}^{1}\right)=L(\gamma)$. This implies

$$
\lim _{n \rightarrow \infty} L\left(P_{n}^{2}\right)=\lim _{n \rightarrow \infty} L\left(P_{n}^{3}\right)=0 .
$$

Without loss of generality we can assume that $P_{n}^{2}$ is not empty for all sufficiently large $n \in \mathbb{N}$. The same reasoning as at the end of the first step shows that this leads to a contradiction. Hence for large $n$ the sets $S_{n}$ have to contain two return points, as we are looking for.

Third Step. In this step the transition from the local version of the theorem to the global one is done. This step is the same as the third step in the proof of Theorem 5.1.

The example in the Introduction shows that the regularity conditions required to prove this theorem are necessary. That is, this theorem is best possible.

\section{Concluding Remarks}

Finally we want to put our work in perspective to related recent work on curve reconstruction. We showed that there exists a global bound on the sampling density such that the curve reconstruction problem is solved by a traveling salesman path or tour, respectively. Obviously this bound is much too demanding for many smooth regions of the curve. That is, locally a much lower sampling density should be sufficient. Amenta et al. [4] concretize the idea of a locally dense sampling using the concept of feature size. The medial axis of a plane curve $\gamma$ is the set of points in the plane which have more than one closest point on $\gamma$. The feature size $f(p)$ of a point $p \in \gamma$ is the distance of $p$ to the closest point on the medial axis. Amenta et al. define sampling density based on a parameter $\varepsilon$ by requiring that each point $p \in \gamma$ has a sample point within distance $\varepsilon f(p)$. Several algorithm with this assumption of sampling density have been developed that provably can reconstruct simple, closed, smooth curves [4]-[6], [11]. There is also an experimental study by Althaus et al. [3] that compares several of these algorithms. For nonsmooth curves this notion of sampling density breaks down, since the medial axis passes through the corner points of the curve. Thus one is required to sample the curve infinitely near the corners to satisfy the sampling condition. 
Many curve reconstruction algorithm are based on picking edges from the Delaunay triangulation. In [10] we show that the same regularity assumptions on the curve are necessary to find the correct reconstruction as a subgraph of the one-skeleton Delaunay triangulation. Dey and Wenger [7] present another algorithm that can reconstruct curves with sharp corners.

We showed that the traveling salesman path can reconstruct simple open curves and the traveling salesman tour can reconstruct simple closed curves. However, we do not give a method to detect only from a sample if a curve is open or closed. Dey et al. [6] give an algorithm that does so in the case of simple smooth curves.

Finally, in general it is NP-hard to compute a traveling salesman path or tour, respectively. Althaus and Mehlhorn [2] show that the traveling salesman path/tour can be computed in polynomial time for dense samples from plane curves, satisfying the regularity conditions we specified in this paper.

\section{Acknowledgments}

I want to thank Emo Welzl and Nicola Galli for helpful discussions.

\section{References}

1. A.D. Aleksandrov, Yu.G. Reshetnyak, Integral Curvature of a Curve in $n$-Dimensional Euclidean Space, Siberian Math. J. 29(1) (1988), 1-16.

2. E. Althaus, K. Mehlhorn, TSP-Based Curve Reconstruction in Polynomial Time, Proc. 11th SIAM Symp. on Discrete Algorithms, pp. 686-695 (2000).

3. E. Althaus, K. Mehlhorn, S. Näher, S. Schirra, Experiments on Curve Reconstruction, Proc. ALENEX 2000 (2000).

4. N. Amenta, M. Bern, D. Eppstein, The Crust and the $\beta$-Skeleton: Combinatorial Curve Reconstruction, Graph. Models Image Process. 60/2(2) (1998), 125-135.

5. T.K. Dey, P. Kumar, A Simple Provable Algorithm for Curve Reconstruction, Proc. 10th SIAM Symp. on Discrete Algorithms, pp. 893-894 (1999).

6. T.K. Dey, K. Mehlhorn, E.A. Ramos, Curve Reconstruction: Connecting Dots with Good Reason, Proc. 15th ACM Symp. on Computational Geometry, pp. 197-206 (1999).

7. T.K. Dey, R. Wenger, Reconstructing Curves with Sharp Corner, Proc. 16th ACM Symp. on Computational Geometry, pp. 233-241 (2000).

8. K.J. Falconer, The Geometry of Fractal Sets, Cambridge University Press, Cambridge (1985).

9. G.B. Folland, Real Analysis, Modern Techniques and Their Applications, 2nd edn., Wiley-Interscience, New York (1999).

10. U. Adamy, J. Giesen, M. John, Surface Reconstruction Using Umbrella Filters, submitted to Comput. Geom. Theory Appl. (2000).

11. C. Gold, Crust and Anti-Crust: A One-Step Boundary and Skeleton Extraction Algorithm, Proc. 15th ACM Symp. on Computational Geometry, pp. 189-196 (1999).

12. K. Menger (ed.), Ergebnisse eines Mathematischen Kolloquiums 2, Kolloquium 5.II.1930, Teubner, Leipzig (1932).

13. K. Menger, Untersuchungen über eine allgemeine Metrik. Vierte Untersuchung. Zur Metrik der Kurven, Math. Ann. 103 (1932), 467-501.

14. Yu.G. Reshetnyak, Some Applications of Integral Geometry to the Theory of Curves of Finite Rotation, Siberian Math. J. 29(1) (1988), 109-116.

Received May 17, 1999, and in revised form February 22, 2000. Online publication August 21, 2000. 